\title{
A Box Particle Filter Method for Tracking Multiple Extended Objects
}

\author{
Allan De Freitas ${ }^{1}$, Lyudmila Mihaylova ${ }^{1, *}$, Amadou Gning ${ }^{2}$, Marek Schikora ${ }^{4}$, Martin Ulmke ${ }^{3}$, Donka Angelova ${ }^{5}$ \\ and Wolfgang Koch ${ }^{3}$
}

\begin{abstract}
Extended objects generate a variable number of multiple measurements. In contrast with point targets, extended objects are characterized with their size or volume, and orientation. Multiple object tracking is a notoriously challenging problem due to complexities caused by data association. This paper develops a box particle filter method for multiple extended object tracking, and for the first time it is shown how interval based approaches can deal efficiently with data association problems and reduce the computational complexity of the data association. The box particle filter relies on the concept of a box particle. A box particle represents a random sample and occupies a controllable rectangular region of non-zero volume in the object state space. A theoretical proof of the generalized likelihood of the box particle filter for multiple extended objects is given based on a binomial expansion. Next the performance of the box particle filter is evaluated using a challenging experiment with the appearance and disappearance of objects within the area of interest, with real laser rangefinder data. The box particle filter is compared with a state-of-the-art particle filter with point particles. Accurate and robust estimates are obtained with the box particle filter, both for the kinematic states and extent parameters, with significant reductions in computational complexity. The box particle filter reduction of computational time is at least $32 \%$ compared with the particle filter working with point particles for the experiment presented. Another advantage of the box particle filter is its robustness to initialization uncertainty.
\end{abstract}

Index Terms - sequential Monte Carlo methods, extended objects, multiple object tracking, non-linear estimation, box particle filter.

\section{INTRODUCTION}

$\mathbf{F}$ OR decades scientists and engineers have focused their attention on finding estimates of states of objects (e.g. the centre of gravity location of an airplane) and their size (in a two dimensional consideration) or their volume (in the three dimensional space). Such extended objects are often considered in intelligent transportation systems (crowds of people [1], a convoy of vehicles [2]), surveillance (ships [3]), robotics (a swarm of unmanned aerial vehicles jointly performing a task), to name a few. There are also results with

1 Department of Automatic Control and Systems Engineering, University of Sheffield, Sheffield, United Kingdom, Email: adefreitas1@sheffield.ac.uk, 1.s.mihaylova@sheffield.ac.uk.

2 School of Engineering and Computer Science, University of Hull, Hull, United Kingdom, Email: e.gning@hull.ac.uk.

3 Department of Sensor Data and Information Fusion, Fraunhofer FKIE, Wachtberg 53343, Germany, Email: wolfgang.koch@fkie.fraunhofer.de, martin.ulmke@fkie.fraunhofer.de

4 Digital Farming, Bayer CropsScience AG, Langenfeld 40764, Germany, Email: marek.schikora@gmail.com

5 Bulgarian Academy of Sciences, Acad. G. Bonchev - 25A, Sofia, Bulgaria, Email: donka@bas.bg

*Corresponding author different types of data: radar [3], image and video [4], laser range sensors [5], LiDAR data (radioactive clouds [6]) and others.

Extended objects are characterized by their size and orientation, in contrast to point objects ([7], [8]) where the whole is approximated with a single point. Extended objects may generate varying numbers of multiple measurements which require efficient data association algorithms. While tracking point objects has been widely studied, and efficient solutions have been developed, the problem of extended object tracking is still challenging and requires new efficient approaches. Moreover, tracking multiple extended objects is not sufficiently studied, except for recent works, e.g. [8]. Extended objects can spawn, merge and cross their trajectories. The methods for extended object tracking can be broadly classified into several categories: random finite set statistics methods (the probabilistic hypothesis density (PHD) filter [9], [10], Cardinality PHD filter [11], multi-Bernoulli Filters [12] etc.), sequential Monte Carlo methods and Markov Chain Monte Carlo methods for Bayesian state space models [7], analytical type of methods [13], [14]. The reader is referred to the surveys [7], [8] where detailed classification of the methods for extended object tracking is given.

Various models for the representation of the shape of an object have been explored. In [3], the shape of an object is modelled as an ellipse and the parameters of the ellipse are directly related to the measurements. In [15] multiple ellipses are considered for the modeling of a single extended object. The concept of a spatial distribution over the object extent was introduced in [16], where the parameters specify the region of the spatial distribution. This concept has also been applied in a track-before-detect setting [17]. In [18], the extent parameter is represented by a random matrix. In [19], the shape of an object is described by an implicit function instead of in a parametric form. Similarly, the shape contour describing the extent is modelled with a Gaussian Process in [20].

In general, when model complexities lead to solutions for state space models without a closed form, particle based approximations are utilized. However, particle based approaches typically suffer due to high computational requirements. This paper proposes a box particle filter (Box PF) method for solving the multiple extended object tracking problem. The Box PF is a combination of interval based techniques and the Bayesian framework. The Box PF for point object state estimation has been used in a number of applications, including localization problems [21] and terrain navigation [22], [23]. The theoretical justification of the point object Box PF 
method is derived in [24], [25]. Filters based on the box concept have been shown to have significant computational efficiency without sacrificing accuracy, robustness to different types of uncertainty in the measurements, and robustness to filter initialization . Preliminary results for the tracking of a single extended object with the Box PF are reported in [26] and tested with simulation data, without a comparison with other approaches. The likelihood function of the Box PF is derived in [27] from geometrical considerations.

The novel contribution of this paper is the development of a Box PF method for multiple extended object tracking with clutter. A theoretical proof of the generalized likelihood is given based on a binomial expansion. An efficient implementation of the generalized Box PF method for multiple extended object tracking in clutter is developed. For the first time it is shown how interval based approaches can be used to deal with data association and to reduce the computational complexity in the data association process. Finally, a comparison with a stateof-the-art PF using point samples and clustering algorithm over a challenging real dataset is presented.

The remainder of this paper is structured in the following manner: Section II gives details of the general problem formulation for multiple extended object tracking. In Section III the new Box PF for multiple extended object tracking is presented. In Section IV an evaluation of the effectiveness of the proposed method is presented and conclusions are drawn in Section V.

\section{Multiple Extended Object Tracking as State AND PARAMETER ESTIMATION}

The multiple extended object tracking problem can be formulated as joint state and parameter estimation in the presence of multiple measurements coming simultaneously from the border or surface of multiple objects. It is also considered that some of the measurements may not originate from an object, in this case referred to as clutter. The latent states of all the objects are combined into a single state vector with fixed dimension, $\boldsymbol{x}_{k}=\left(\boldsymbol{x}_{1, k}^{\top}, \boldsymbol{x}_{2, k}^{\top}, \ldots, \boldsymbol{x}_{N_{T}, k}^{\top}\right)^{\top}, N_{T}$ represents the maximum number of extended objects, and $k \in\{1,2 \ldots T\}$ the discrete time steps, with $T$ representing the final time step. The notation $(\cdot)^{\top}$ denotes the transpose operator.

In extended object tracking, each extended object sub-state vector is defined as $\boldsymbol{x}_{i, k}=\left(\boldsymbol{X}_{i, k}^{\top}, \boldsymbol{\Theta}_{i, k}^{\top}\right)^{\top}$. The subset of states, $\boldsymbol{X}_{i, k}$, comprises all the states related to the kinematics (e.g. position coordinates, velocities) of the centroid of motion of the object. This typically includes the position, velocity and any other higher order position derivatives defined by the motion model. The subset of states, $\boldsymbol{\Theta}_{i, k}$, comprises all the parameters used to model the extent of the object. This allows for the extent of the object to be represented by a variety of parametric shapes. It is assumed that the kinematic states and parameter states are independent.

At each time step, $k$, an unordered set of measurements is collected, $\boldsymbol{Z}_{k}=\left\{\boldsymbol{z}_{1, k}, \boldsymbol{z}_{2, k} \ldots \boldsymbol{z}_{M_{k}, k}\right\}$, where $M_{k}=$ $\sum_{i}^{N_{T}} M_{T, k}^{i}+M_{C, k}$ represents the total number of measurements at time step $k$. The number of measurements $M_{T, k}^{i}$ originating from the visible border or surface of the source is considered as a Poisson-distributed random variable with a mean value $\lambda_{T, i}$, i.e., $M_{T, k}^{i} \sim \operatorname{Poisson}\left(\lambda_{T, i}\right)$. Similarly, the number of clutter measurements is $M_{C, k} \sim \operatorname{Poisson}\left(\lambda_{C}\right)$, where $\lambda_{C}$ is the mean value of the clutter measurements.

\section{A. Birth and Disappearance of Extended Objects}

In multiple object tracking, an object may enter or leave the area observed by the sensors at any time. This is referred to as the birth or death of an object, respectively. To cater for a varying number of extended objects, a binary variable representing the existence of each extended object is introduced, inspired by [28], [29], $\boldsymbol{e}_{k}=\left(e_{1, k}, e_{2, k} \ldots e_{N_{T}, k}\right)^{\top}$ with $e_{i, k} \in\{0,1\}$, where the values of 0 and 1 correspond to a non-existent and existent object, respectively. The existence variable, $e_{i, k}$, evolves according to a Markov chain with the following property,

$$
p\left(e_{i, k} \mid e_{i, k-1}=\ell\right)= \begin{cases}P_{e} & \text { when } e_{i, k}=\ell, \\ 1-P_{e} & \text { otherwise }\end{cases}
$$

where $P_{e}$ represents the probability of existence.

\section{B. Problem Formulation within the Bayesian Framework}

It is required to estimate $\boldsymbol{x}_{k}$ and $\boldsymbol{e}_{k}$, jointly considered as $\boldsymbol{s}_{k}=\left(\boldsymbol{x}_{k}^{\top}, \boldsymbol{e}_{k}^{\top}\right)^{\top}$. Suppose that the initial probability density function (pdf) of $p\left(\boldsymbol{s}_{0}\right)$ is given. According to the Bayesian framework the posterior state pdf given the sensor measurements, $p\left(\boldsymbol{s}_{k} \mid \boldsymbol{Z}_{1: k}\right)$, where $\boldsymbol{Z}_{1: k}=\left\{\boldsymbol{Z}_{1}, \ldots, \boldsymbol{Z}_{k}\right\}$, is of interest. The posterior state pdf can be updated iteratively through two steps. A prediction step

$$
p\left(\boldsymbol{s}_{k} \mid \boldsymbol{Z}_{1: k-1}\right)=\int p\left(\boldsymbol{s}_{k} \mid \boldsymbol{s}_{k-1}\right) p\left(\boldsymbol{s}_{k-1} \mid \boldsymbol{Z}_{1: k-1}\right) d \boldsymbol{s}_{k-1},
$$

followed by an update step

$$
p\left(\boldsymbol{s}_{k} \mid \boldsymbol{Z}_{1: k}\right)=\frac{p\left(\boldsymbol{Z}_{k} \mid \boldsymbol{s}_{k}\right) p\left(\boldsymbol{s}_{k} \mid \boldsymbol{Z}_{1: k-1}\right)}{p\left(\boldsymbol{Z}_{k} \mid \boldsymbol{Z}_{1: k-1}\right)},
$$

where $p\left(\boldsymbol{s}_{k} \mid \boldsymbol{Z}_{1: k-1}\right)$ is the predictive posterior state pdf, $p\left(\boldsymbol{s}_{k} \mid \boldsymbol{s}_{k-1}\right)$ is the state transition pdf, $p\left(\boldsymbol{Z}_{k} \mid \boldsymbol{s}_{k}\right)$ is the likelihood function and $p\left(\boldsymbol{Z}_{k} \mid \boldsymbol{Z}_{1: k-1}\right)$ is a normalization factor.

\section{State Transition Representation}

The state transition pdf can be further factorized as:

$$
p\left(\boldsymbol{s}_{k} \mid \boldsymbol{s}_{k-1}\right)=\prod_{i=1}^{N_{T}} p\left(\boldsymbol{x}_{i, k} \mid \boldsymbol{x}_{i, k-1}, e_{i, k}, e_{i, k-1}\right) p\left(e_{i, k} \mid e_{i, k-1}\right) .
$$

The sub-state transition pdf for the $i$ th object is defined as:

$$
\begin{aligned}
p\left(\boldsymbol{x}_{i, k} \mid \boldsymbol{x}_{i, k-1}, e_{i, k}, e_{i, k-1}\right)= & \left\{\begin{array}{lr}
p_{b}\left(\boldsymbol{x}_{i, k}\right) & \left\{e_{i, k}, e_{i, k-1}\right\}=\{1,0\}, \\
p_{d}\left(\boldsymbol{x}_{i, k}\right) & \left\{e_{i, k}\right\}=\{0\}, \\
p\left(\boldsymbol{x}_{i, k} \mid \boldsymbol{x}_{i, k-1}\right) & \left\{e_{i, k}, e_{i, k-1}\right\}=\{1,1\},
\end{array}\right.
\end{aligned}
$$

where $p_{b}\left(\boldsymbol{x}_{i, k}\right)$ and $p_{d}\left(\boldsymbol{x}_{i, k}\right)$ are the probability values of an object birth and death respectively, and $p\left(\boldsymbol{x}_{i, k} \mid \boldsymbol{x}_{i, k-1}\right)$ represents the motion of existent extended objects. 


\section{Likelihood Representation}

The likelihood in equation (3) can be calculated in various ways with different data association algorithms. One of the best approaches, which alleviates the combinatorial complexity in data association, is proposed in [30]. It adopts Poisson assumptions for the number of measurements originating from the objects and the number of clutter points. This generalized likelihood function is of the form

$$
p\left(\boldsymbol{Z}_{k} \mid \boldsymbol{s}_{k}\right)=\frac{e^{-\sum_{i \in \mathcal{I}} \lambda_{T, i}}}{M_{k} !} \prod_{m=1}^{M_{k}}\left(\rho+\sum_{i \in \mathcal{I}} \lambda_{T, i} p\left(\boldsymbol{z}_{k}^{m} \mid \boldsymbol{x}_{i, k}\right)\right),
$$

where $\mathcal{I}$ denotes a set corresponding to the index of active targets at the current time step, $\lambda_{C}$ and $\lambda_{T, i}$ are the mean values for the Poisson distribution describing the number of measurements originating from clutter and the $i$ th target respectively, $\rho=\frac{\lambda_{C}}{A_{C}}$ is the clutter density, $A_{C}$ represents the area of the region where clutter may be emitted from, and $p\left(\boldsymbol{z}_{k}^{m} \mid \boldsymbol{x}_{i, k}\right)$ is the measurement likelihood for a single object.

Consider the use of multiple sensors to observe the extended objects. The state of sensor $s$ is known and given by $\boldsymbol{x}_{s, k}=\left(x_{s, k}, y_{s, k}, \alpha_{1, k}, \alpha_{2, k}\right)^{\top}$, where $\left(x_{s, k}, y_{s, k}\right)$ are the sensor position coordinates, $\alpha_{1, k}$ and $\alpha_{2, k}$ represent two parameters defining the angle of view of the sensor. When an extended object is visible from sensor $s$, the sensor states and object system sub-states geometrically define the visible border of the extended object, $\mathcal{V}_{k}\left(\boldsymbol{x}_{i, k}, \boldsymbol{x}_{s, k}\right)$. For a single time instance $k$, measurement $\boldsymbol{z}_{k}^{m}$ is related to a specific point on the visible surface of an extended object. This point is referred to as the $m$ th point source and denoted by $\boldsymbol{V}_{i, k}^{m}$.

The relationship between $\boldsymbol{z}_{k}^{m}$ and $\boldsymbol{V}_{i, k}^{m}$ is given by

$$
\boldsymbol{z}_{k}^{m}=h\left(\boldsymbol{V}_{i, k}^{m}\right)+\boldsymbol{w}_{k}^{m},
$$

where $h(\cdot)$ is a non-linear function, and the measurement noise $\boldsymbol{w}_{k}^{m}$ is assumed (but not restricted) to be white zero mean Gaussian, with a known covariance matrix $\Sigma$.

The measurement likelihood for a single object consists of the combination of two pdfs,

$$
p\left(\boldsymbol{z}_{k}^{m} \mid \boldsymbol{x}_{i, k}\right)=\int p\left(\boldsymbol{z}_{k}^{m} \mid \boldsymbol{V}_{i, k}^{m}\right) p\left(\boldsymbol{V}_{i, k}^{m} \mid \boldsymbol{x}_{i, k}\right) d \boldsymbol{V}_{i, k}^{m},
$$

where $p\left(\boldsymbol{z}_{k}^{m} \mid \boldsymbol{V}_{i, k}^{m}\right)$ denotes the likelihood of the measurement given a point source (based on (7)), and $p\left(\boldsymbol{V}_{i, k}^{m} \mid \boldsymbol{x}_{i, k}\right)$ is the likelihood of the point source given the object sub-states.

One simplified assumption about the distribution of the point sources of measurements, given the object sub-states and the sensor states, is a uniform distribution along the region $\mathcal{V}_{k}\left(\boldsymbol{x}_{i, k}, \boldsymbol{x}_{s, k}\right)$, visible from the sensor position, i.e.

$$
p\left(\boldsymbol{V}_{i, k}^{j} \mid \boldsymbol{x}_{i, k}\right)=\mathcal{U}_{\mathcal{V}_{k}\left(\boldsymbol{x}_{i, k}, \boldsymbol{x}_{s, k}\right)}\left(\boldsymbol{V}_{k}\right)=\frac{1}{\left\|\mathcal{V}_{k}\left(\boldsymbol{x}_{i, k}, \boldsymbol{x}_{s, k}\right)\right\|},
$$

where $\mathcal{U}_{\mathcal{V}_{k}\left(\boldsymbol{x}_{i, k}, \boldsymbol{x}_{s, k}\right)}(\cdot)$ is a uniform pdf with the support $\mathcal{V}_{k}\left(\boldsymbol{x}_{i, k}, \boldsymbol{x}_{s, k}\right)$ and $\left\|\mathcal{V}_{k}\left(\boldsymbol{x}_{i, k}, \boldsymbol{x}_{s, k}\right)\right\|$ denotes some measure of the region $\mathcal{V}_{k}\left(\boldsymbol{x}_{i, k}, \boldsymbol{x}_{s, k}\right)$, such as the Euclidean norm.

\section{Derivation of the New Extended Object Box PARTICLE FILTER}

No closed form solution exists for the prediction and updating of the posterior state pdf in equations (2) and (3) due to complexities in the state space model. Therefore, Monte Carlo methods that approximate the posterior state pdf are considered. The Box PF is a Monte Carlo method based on the recently emerged concept of "generalized boxes", also called "box particles" [31]. A box is a controllable rectangular region having a non-zero volume in the state space. The main idea of the Box PF is to replace the point particles in general PF algorithms with region-particles, i.e. with box particles.

\section{A. Time Update Step of the Box Particle Filter}

The Box PF represents the posterior pdf by a weighted summation of uniform distributions,

$$
p\left(\boldsymbol{s}_{k-1} \mid \boldsymbol{Z}_{1: k-1}\right) \approx \sum_{p=1}^{N} w_{k-1}^{(p)} U_{\left[\boldsymbol{s}_{k-1}^{(p)}\right]}\left(\boldsymbol{s}_{k-1}\right),
$$

where the notation $U_{[\cdot]}(x)$ denotes a uniform distribution with support $^{1}[\cdot]$, corresponding to a box particle, $N$ is the number of box particles, and $w_{k-1}^{(p)}$ is the normalized weight for box particle $p$. The Box PF is suitable for various types of uncertainties in the measurements: i.e. interval, stochastic and data association uncertainties as shown in [32]. In this application, the dimensions of $s_{k}$ corresponding to the existence vector, $\boldsymbol{e}_{k}$, are still considered to have zero volume.

The Box PF framework is based on the prediction and update steps as presented by equations (2) and (3). For the Box PF, the prediction step can be written as:

$$
\begin{aligned}
p\left(\boldsymbol{s}_{k} \mid \boldsymbol{Z}_{1: k-1}\right) & \approx \int p\left(\boldsymbol{s}_{k} \mid \boldsymbol{s}_{k-1}\right) \sum_{p=1}^{N} w_{k-1}^{(p)} U_{\left[\boldsymbol{s}_{k-1}^{(p)}\right]}\left(\boldsymbol{s}_{k-1}\right) d \boldsymbol{s}_{k-1} \\
& =\sum_{p=1}^{N} w_{k-1}^{(p)} \int_{\left[\boldsymbol{s}_{k-1}^{(p)}\right]} p\left(\boldsymbol{s}_{k} \mid \boldsymbol{s}_{k-1}\right) U_{\left[\boldsymbol{s}_{k-1}^{(p)}\right]}\left(\boldsymbol{s}_{k-1}\right) d \boldsymbol{s}_{k-1} .
\end{aligned}
$$

Representing the transition pdf in equation (4) as a transition function, $f$, an inclusion function $[f]$ (see [25] for more details) where $f([s]) \subseteq[f]([s])$ can be obtained. For the inclusion function, with $\forall p=1, \ldots, N$, if $\boldsymbol{s}_{k-1} \in\left[\boldsymbol{s}_{k-1}^{(p)}\right]$ then $\boldsymbol{s}_{k} \in[f]\left(\left[\boldsymbol{s}_{k-1}^{(p)}\right]\right)$. Thus, for all $p=1, \ldots, N$

$$
p\left(\boldsymbol{s}_{k} \mid \boldsymbol{s}_{k-1}\right) U_{\left[\boldsymbol{s}_{k-1}^{(p)}\right]}\left(\boldsymbol{s}_{k-1}\right)=0, \forall \boldsymbol{s}_{k} \notin[f]\left(\left[\boldsymbol{s}_{k-1}^{(p)}\right]\right) .
$$

Using interval analysis techniques, the support of the function for the pdf terms in (11) can be approximated by $[f]\left(\left[\boldsymbol{s}_{k-1}^{(p)}\right]\right)$. In the Box PF algorithm each pdf term in (11) is approximated by one uniform pdf component having as support the interval $[f]\left(\left[\boldsymbol{s}_{k-1}^{(p)}\right]\right)$, i.e.,

$$
\int_{\left[\mathbf{s}_{k-1}^{(p)}\right]} p\left(\boldsymbol{s}_{k} \mid \boldsymbol{s}_{k-1}\right) U_{\left[\boldsymbol{s}_{k-1}^{(p)}\right]}\left(\boldsymbol{s}_{k-1}\right) d \boldsymbol{s}_{k-1} \approx U_{[f]\left(\left[\boldsymbol{s}_{k-1}^{(p)}\right]\right)}\left(\boldsymbol{s}_{k}\right) .
$$

Combining (11) and (13) gives

$$
\begin{aligned}
p\left(\boldsymbol{s}_{k} \mid \boldsymbol{Z}_{1: k-1}\right) & \approx \sum_{p=1}^{N} w_{k-1}^{(p)} U_{[f]\left(\left[\boldsymbol{s}_{k-1}^{(p)}\right]\right)}\left(\boldsymbol{s}_{k}\right) \\
& =\sum_{p=1}^{N} w_{k-1}^{(p)} U_{\left[\boldsymbol{s}_{k \mid k-1}^{(p)}\right]}\left(\boldsymbol{s}_{k}\right) .
\end{aligned}
$$

${ }^{1}$ The support of a function is the set of points where the function is not zero-valued or, in the case of functions defined on a topological space, the closure of that set. 
Approximating each pdf term in equation (14) using one uniform pdf component may not be accurate enough. However, as for the PF, it is sufficient to approximate the first moments of the pdf. If a more accurate representation is required then each term can be approximated as a mixture of uniform pdfs as shown in [25].

\section{B. Box Particle Filter Likelihood for Multiple Extended Ob- jects}

A reasonable assumption for the Box PF is that the likelihood of a measurement given a point source has a bounded support. This leads to the definition of an interval measurement, $\left[\boldsymbol{z}_{k}^{m}\right]$, and corresponding likelihood approximated by a uniform distribution, i.e. $p\left(\boldsymbol{z}_{k}^{m} \mid \boldsymbol{V}_{i, k}^{m}\right)=U_{\left[\boldsymbol{z}_{k}^{m}\right]}\left(h\left(\boldsymbol{V}_{i, k}^{m}\right)\right)$.

Brute Force Approach: As in the standard PF, the update step for the Box PF assigns a weighting to each of the predicted box particles. However, it is also required to apply an interval technique, called contraction [33], to each box particle. Contraction is used to eliminate regions of the predicted box particles which are not consistent with the object emitted measurements. This is a challenging task when dealing with extended objects and clutter. To define the weight updates and contraction, it is required to derive an expression for the posterior state pdf.

Proposition 1: An alternative form of the generalized likelihood function of equation (6) is given by,

$$
\begin{aligned}
& p\left(\boldsymbol{Z}_{k} \mid \boldsymbol{s}_{k}\right)=\frac{e^{-\sum_{i \in \mathcal{I}} \lambda_{T, i}}}{M_{k} !}\left(\rho^{M_{k}}\right. \\
& \left.+\sum_{m=1}^{M_{k}} \sum_{j=1}^{\left({ }^{M_{k}}{ }^{m}\right.} \sum_{n=1}^{|\mathcal{I}|^{m}} \rho^{M_{k}-m} \prod_{\ell=1}^{m} \lambda_{T,\left(\boldsymbol{b}_{m, n}\right)_{\ell}} p\left(\boldsymbol{z}_{k}^{\left(\boldsymbol{a}_{m, j}\right)_{\ell}} \mid \boldsymbol{x}_{\left(\boldsymbol{b}_{m, n}\right)_{\ell}, k}\right)\right),
\end{aligned}
$$

where the notation $|\cdot|$ denotes the cardinality of a set, $\left(\left(\boldsymbol{a}_{m, j}\right)_{m=1}^{M_{k}}\right)_{j=1}^{\left(\begin{array}{c}M_{k} \\ m\end{array}\right)}$ is a sequence of sequences corresponding to the index for all combinations of measurements, and $\left(\left(\boldsymbol{b}_{m, n}\right)_{m=1}^{M_{k}}\right)_{n=1}^{|\mathcal{I}|^{m}}$ is a sequence of sequences corresponding to the index for all existent object to measurement associations. Proof: See Appendix A.

Example: consider a state vector for the case of when there are a maximum of three extended objects, where currently only the first and third objects are existent, i.e. $\mathcal{I}=\{1,3\}$, with two measurements. The sequences are thus defined as: $\left(\boldsymbol{a}_{1,1}\right)=(1) ;\left(\boldsymbol{a}_{1,2}\right)=(2) ;\left(\boldsymbol{a}_{2,1}\right)=(1,2) ;\left(\boldsymbol{b}_{1,1}\right)=(1)$; $\left(\boldsymbol{b}_{1,2}\right)=(3) ;\left(\boldsymbol{b}_{2,1}\right)=(1,1) ;\left(\boldsymbol{b}_{2,2}\right)=(1,3) ;\left(\boldsymbol{b}_{2,3}\right)=(3,1)$ $\left(\boldsymbol{b}_{2,4}\right)=(3,3)$, resulting in the following generalized likelihood expression:

$$
\begin{aligned}
& p\left(\boldsymbol{Z}_{k} \mid \boldsymbol{s}_{k}\right)= \\
& \frac{e^{-\left(\lambda_{T, 1}+\lambda_{T, 3}\right)}}{2 !}\left(\rho^{2}+\rho \lambda_{T, 1} p\left(\boldsymbol{z}_{k}^{1} \mid \boldsymbol{x}_{1, k}\right)+\rho \lambda_{T, 3} p\left(\boldsymbol{z}_{k}^{1} \mid \boldsymbol{x}_{3, k}\right)+\right. \\
& \rho \lambda_{T, 1} p\left(\boldsymbol{z}_{k}^{2} \mid \boldsymbol{x}_{1, k}\right)+\rho \lambda_{T, 3} p\left(\boldsymbol{z}_{k}^{2} \mid \boldsymbol{x}_{3, k}\right)+ \\
& \lambda_{T, 1}^{2} p\left(\boldsymbol{z}_{k}^{1} \mid \boldsymbol{x}_{1, k}\right) p\left(\boldsymbol{z}_{k}^{2} \mid \boldsymbol{x}_{1, k}\right)+\lambda_{T, 1} \lambda_{T, 3} p\left(\boldsymbol{z}_{k}^{1} \mid \boldsymbol{x}_{1, k}\right) p\left(\boldsymbol{z}_{k}^{2} \mid \boldsymbol{x}_{3, k}\right)+ \\
& \left.\lambda_{T, 3} \lambda_{T, 1} p\left(\boldsymbol{z}_{k}^{1} \mid \boldsymbol{x}_{3, k}\right) p\left(\boldsymbol{z}_{k}^{2} \mid \boldsymbol{x}_{1, k}\right)+\lambda_{T, 3}^{2} p\left(\boldsymbol{z}_{k}^{1} \mid \boldsymbol{x}_{3, k}\right) p\left(\boldsymbol{z}_{k}^{2} \mid \boldsymbol{x}_{3, k}\right)\right) .
\end{aligned}
$$

This example highlights the fact that the evaluation of the generalized likelihood for a single state results in a summation of terms. Each term corresponds to a unique measurement association and for each object assigned measurement, an association with a specific object.

The posterior state pdf can be obtained through the combination of the predictive posterior state pdf and generalized likelihood:

$$
\begin{aligned}
p\left(\boldsymbol{s}_{k} \mid \boldsymbol{Z}_{1: k}\right) & =\frac{1}{\alpha_{k}} p\left(\boldsymbol{Z}_{k} \mid \boldsymbol{s}_{k}\right) p\left(\boldsymbol{s}_{k} \mid \boldsymbol{Z}_{1: k-1}\right) \\
& =\sum_{p=1}^{N} \frac{w_{k-1}^{(p)} e^{-\sum_{i \in \mathcal{I}}(p) \lambda_{T, i}}}{\alpha_{k} M_{k} !}\left(\rho^{M_{k}} U_{\left[\boldsymbol{s}_{k \mid k-1}^{(p)}\right]}\left(\boldsymbol{s}_{k}\right)+\right. \\
& \sum_{m=1}^{M_{k}} \sum_{j=1}^{\left({ }^{M_{k}}{ }_{m}^{m}\right)^{\left|\mathcal{I}^{(p)}\right|^{m}}} \sum_{n=1}^{m} \rho^{M_{k}-m} \prod_{\ell=1}^{m} \lambda_{T,\left(\boldsymbol{b}_{m, n}\right)_{\ell} \times} \times \\
& \left.p\left(\boldsymbol{z}_{k}^{\left(\boldsymbol{a}_{m, j}\right)_{\ell}} \mid\left[\boldsymbol{x}_{\left(\boldsymbol{b}_{m, n}\right)_{\ell}, k \mid k-1}^{(p)}\right]\right) U_{\left[\boldsymbol{s}_{k \mid k-1}^{(p)}\right]}\left(\boldsymbol{s}_{k}\right)\right)
\end{aligned}
$$

The expressions in the product of each of the latter terms can be further reduced based on the decomposition of the measurement likelihood for a single object, i.e. equation (8). For notational convenience, $\left(\boldsymbol{a}_{m, j}\right)_{\ell}$ and $\left(\boldsymbol{b}_{m, n}\right)_{\ell}$ are represented by $a_{\ell}$ and $b_{\ell}$ respectively,

$$
\begin{aligned}
& p\left(\boldsymbol{z}_{k}^{a_{\ell}} \mid\left[\boldsymbol{x}_{b_{\ell}, k}^{(p)}\right]\right) U_{\left[\boldsymbol{s}_{k \mid k-1}^{(p)}\right]}\left(\boldsymbol{s}_{k}\right)= \\
& \quad \int U_{\left[\boldsymbol{s}_{k \mid k-1}^{(p)}\right]}\left(\boldsymbol{s}_{k}\right) U_{\left[\boldsymbol{z}_{k}^{\left.a_{\ell}\right]}\right]}\left(h\left(\boldsymbol{V}_{b_{\ell}, k}^{a_{\ell}}\right)\right) \mathcal{U}_{\mathcal{V}_{k}\left(\boldsymbol{x}_{b_{\ell}, k}, \boldsymbol{x}_{s, k}\right)}\left(\boldsymbol{V}_{b_{\ell}, k}^{a_{\ell}}\right) d \boldsymbol{V}_{b_{\ell}, k}^{a_{\ell}} .
\end{aligned}
$$

The terms within the integration form a constant function with a support being the following region

$S_{p}^{a_{\ell}, b_{\ell}}=\left\{\boldsymbol{s}_{k} \in\left[\boldsymbol{s}_{k \mid k-1}^{(p)}\right] \mid \boldsymbol{V}_{b_{\ell}, k}^{a_{\ell}} \in \mathcal{V}_{k}\left(\boldsymbol{x}_{b_{\ell}, k}, \boldsymbol{x}_{s, k}\right), h\left(\boldsymbol{V}_{b_{\ell}, k}^{a_{\ell}}\right) \in\left[\boldsymbol{z}_{k}^{a_{\ell}}\right]\right\}$.

This represents a constraint and from its expression it can be deduced that the predicted supports $\left[\boldsymbol{s}_{k \mid k-1}^{(p)}\right]$, from the time update pdf approximation, have to be contracted with respect to the interval measurements $\left[\boldsymbol{Z}_{k}\right]$. This forms the basis for what is known as a constraints satisfaction problem (CSP). In this paper the Constraints Propagation (CP) technique [33] is used to contract the box particles.

The contracted box particle is represented by $\left[\boldsymbol{s}_{k}^{a_{\ell},(p)}\right]$. It is important to note that contraction only occurs on the sub-states corresponding to the object indexed by $b_{\ell}$, i.e. $\left[\boldsymbol{s}_{k}^{a_{\ell},(p)}\right]=$ $\left(\left[\boldsymbol{x}_{1, k \mid k-1}^{(p)}\right], \ldots,\left[\boldsymbol{x}_{b_{\ell}, k}^{a_{\ell},(p)}\right], \ldots,\left[\boldsymbol{x}_{N_{T}, k \mid k-1}^{(p)}\right],\left[\boldsymbol{e}_{k \mid k-1}^{(p)}\right]\right)^{\top} \quad$ where $\left[\boldsymbol{x}_{i, k}^{a_{\ell},(p)}\right]$ represents the sub-states of object $i$ contracted by the measurement indexed by $a_{\ell}$. Following the definition of the set $S_{p}^{a_{\ell}, b_{\ell}}$ in equation (19), equation (18) can be rewritten as follows

$p\left(\boldsymbol{z}_{k}^{a_{\ell}} \mid\left[\boldsymbol{x}_{b_{\ell}, k}^{(p)}\right]\right) U_{\left[\boldsymbol{s}_{k \mid k-1}^{(p)}\right]}\left(\boldsymbol{s}_{k}\right)=\frac{\left|\left[\boldsymbol{s}_{k}^{a_{\ell},(p)}\right]\right|}{\left|\left[\boldsymbol{s}_{k \mid k-1}^{(p)}\right]\right|} U_{\left[\boldsymbol{s}_{k}^{a_{\ell},(p)}\right]}\left(\boldsymbol{s}_{k}\right) p\left(\boldsymbol{z}_{k}^{a_{\ell}} \mid\left[\boldsymbol{x}_{b_{\ell}, k}^{(p)}\right]\right)$.

Note, the notation $|\cdot|$ for a box denotes the interval length (respectively the box volume in the multidimensional case), in contrast to the cardinality of a set. If the entire product is considered, $\prod_{\ell=1}^{m} p\left(\boldsymbol{z}_{k}^{a_{\ell}} \mid\left[\boldsymbol{x}_{b_{\ell}, k}^{(p)}\right]\right) U_{\left[\boldsymbol{s}_{k \mid k-1}^{(p)}\right]}\left(\boldsymbol{s}_{k}\right)$, the contracted box, $U_{\left[\boldsymbol{s}_{k}^{a_{1},(p)}\right]}\left(s_{k}\right)$, is further contracted in the same manner as described by equations (19) and (20) by each measurement likelihood for a single object. In terms of interval arithmetic, the product of contracted boxes is equivalent to the intersection 
of the box particles contracted by the individual measurements,

$$
\begin{aligned}
& \prod_{\ell=1}^{m} p\left(\boldsymbol{z}_{k}^{a_{\ell}} \mid\left[\boldsymbol{x}_{b_{\ell}, k}^{(p)}\right]\right) U_{\left[\boldsymbol{s}_{k \mid k-1}^{(p)}\right]}\left(\boldsymbol{s}_{k}\right)= \\
& \frac{\left|\left[\boldsymbol{s}_{k}^{a,(p)}\right]\right|}{\left|\left[\boldsymbol{s}_{k \mid k-1}^{(p)}\right]\right|} U_{\left[\boldsymbol{s}_{k}^{a,(p)}\right]}\left(\boldsymbol{s}_{k}\right) \prod_{\ell=1}^{m} p\left(\boldsymbol{z}_{k}^{a_{\ell}} \mid\left[\boldsymbol{x}_{b_{\ell}, k}^{(p)}\right]\right),
\end{aligned}
$$

where $\left[s_{k}^{a,(p)}\right]=\bigcap_{\ell=1}^{m}\left[s_{k}^{a_{\ell},(p)}\right]$. This results in the following reduced form of the posterior state pdf in (17),

$$
\begin{aligned}
p\left(\boldsymbol{s}_{k} \mid \boldsymbol{Z}_{1: k}\right) & =\sum_{p=1}^{N} \frac{w_{k-1}^{(p)} e^{-\sum_{i \in \mathcal{I}}(p) \lambda_{T, i}}}{\alpha_{k} M_{k} !}\left(\rho^{M_{k}} U_{\left[\boldsymbol{s}_{k \mid k-1}^{(p)}\right]}\left(\boldsymbol{s}_{k}\right)+\right. \\
& \sum_{m=1}^{M_{k}} \sum_{j=1}^{\left(\begin{array}{c}
M_{k} \\
m
\end{array}\right)} \sum_{n=1}^{\left|\mathcal{I}^{(p)}\right|^{m}} \frac{\left|\left[\boldsymbol{s}_{k}^{\boldsymbol{a}_{m, j},(p, n)}\right]\right| \rho^{M_{k}-m}}{\left|\left[\boldsymbol{s}_{k \mid k-1}^{(p)}\right]\right|} \times \\
& \left.U_{\left[\boldsymbol{s}_{k} \boldsymbol{a}_{m, j},(p, n)\right.}\left(\boldsymbol{s}_{k}\right) \prod_{\ell=1}^{m} \lambda_{T,\left(\boldsymbol{b}_{m, n}\right)_{\ell}} p\left(\boldsymbol{z}_{k}^{\left(\boldsymbol{a}_{m, j}\right)_{\ell}} \mid\left[\boldsymbol{x}_{\left(\boldsymbol{b}_{m, n}\right)_{\ell}, k}^{(p)}\right]\right)\right) .
\end{aligned}
$$

This is a brute force approach which considers every possible measurement association. It is clear from the indices of the summations that a single predicted box particle can result in a summation of a large number of terms. For example, in the case of 3 objects and 15 measurements, each predicted box particle would result in over 1 billion weighted boxes after the update. Thus, the brute force implementation is not computationally tractable.

Standard Approach: There are two causes for why such a considerable number of boxes exists. The first cause is the uncertainty in which measurements are from which objects. This uncertainty can be reduced through the introduction of clustering. The clustering algorithm assigns the index of each measurement to a single cluster set $\mathcal{C}_{i}$, where $i \in 1, \ldots, N_{c}$, with $N_{c}$ the total number of clusters, assumed unknown. Measurements which are close to each other, according to a specific metric, are assigned to the same cluster. The validity of utilizing clustering is based on the assumption that measurements from a single object are typically located within the vicinity of each other in the measurement space. However, care is taken to ensure that the algorithm is robust to sub-optimal clustering. Employing clustering, results in the following approximation of the posterior state pdf:

$$
\begin{aligned}
& p\left(\boldsymbol{s}_{k} \mid \boldsymbol{Z}_{1: k}\right) \approx \sum_{p=1}^{N} \frac{w_{k-1}^{(p)} e^{-\sum_{i \in \mathcal{I}(p) \lambda_{T, i}}}}{\alpha_{k} M_{k} !}\left(\rho^{M_{k}} U_{\left[\boldsymbol{s}_{k \mid k-1}^{(p)}\right]}\left(\boldsymbol{s}_{k}\right)+\right. \\
& \sum_{m=1}^{M_{k}} \sum_{j=1}^{\left(\begin{array}{c}
M_{k} \\
m
\end{array}\right)} \sum_{n=1}^{\left|\mathcal{I}^{(p)}\right|^{d_{j}}} \frac{\left|\left[\boldsymbol{s}_{k}^{\boldsymbol{a}_{m, j},(p, n)}\right]\right| \rho^{M_{k}-m}}{\left|\left[\boldsymbol{s}_{k \mid k-1}^{(p)}\right]\right|} \times \\
& \left.U_{\left[\boldsymbol{s}_{k}\right.}^{\boldsymbol{a}_{m, j},(p, n)_{]}}\left(\boldsymbol{s}_{k}\right) \prod_{\ell=1}^{m} \lambda_{T,\left(\boldsymbol{b}_{m, n}\right)_{\ell}} p\left(\boldsymbol{z}_{k}^{\left(\boldsymbol{a}_{m, j}\right)_{\ell}} \mid\left[\boldsymbol{x}_{\left(\boldsymbol{b}_{m, n}\right)_{\ell}, k}^{(p)}\right]\right)\right),
\end{aligned}
$$

where $d_{j}$ is the number of clusters that the $j$ th unique combination of object assigned measurements originates from, and the sequences $\left(\boldsymbol{b}_{m, n}\right)$ are reduced to only consider the measurements to object associations where measurements from the same cluster are assigned to the same object. Considering the same example of 3 objects and 15 measurements, if the clustering algorithm results in 3 clusters, each indexing 5 of the measurements, the number of weighted box particles after the update per predicted box particle is reduced from over

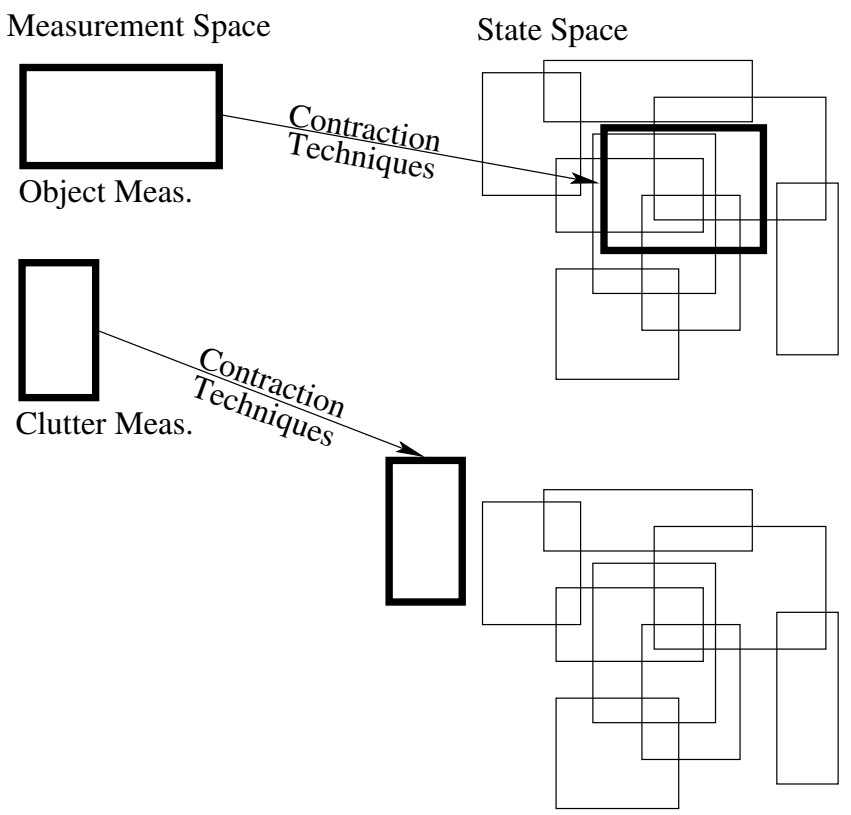

Fig. 1. Illustration of the consistency between a set of box particles and object or clutter measurents.

1 billion to 830584 . Although this reduces the number of weighted boxes by orders of magnitude for each box particle, this still results in a large computational burden.

Interval Analysis Approach: The second cause for the large number of boxes is the uncertainty in which measurements are emitted by an object or are clutter. Using an interval based approach, it is possible to reduce the number of boxes due to this uncertainty.

The weight of each term in the posterior state pdf describes how likely the associations are, given the measurements. As observed in equation (21), each term is non-zero on the predicted state interval contracted by all the assigned object measurements. This interval is equivalent to the intersection of the contraction results for each of the measurements assigned as object originated. Each term can have clutter measurements assigned as object measurements. However, the contraction due to a clutter measurement can be an interval which does not exist, or is disjoint with the contracted intervals from the object originated measurements, as illustrated in Fig. 1. Since the overall result is dependent on the intersection, even a single clutter measurement assigned as an object measurement may result in the corresponding term having a zero weight. The computation of these terms can be avoided by approximating the intersection with the relaxed intersection. The relaxed intersection, first introduced in [34], corresponds to the classical intersection between intervals with the exception that it is allowed to relax a certain number of intervals in order to avoid an empty intersection.

Utilizing the relaxed intersection, the following approxima- 
tion for the posterior state pdf is obtained,

$$
\begin{aligned}
& p\left(\boldsymbol{s}_{k} \mid \boldsymbol{Z}_{1: k}\right) \approx \sum_{p=1}^{N} \frac{w_{k-1}^{(p)} e^{-\sum_{i \in \mathcal{I}^{(p)} \lambda_{T, i}}}\left(\sum_{n=1}^{\left|\mathcal{I}^{(p)}\right|} \frac{\left|\left[\boldsymbol{s}_{k}^{d,(p, n)}\right]\right| \rho^{M_{k}-u^{(p)}}}{\alpha_{k} M_{k} !}\right.}{\left|\left[\boldsymbol{s}_{k \mid k-1}^{(p)}\right]\right|} \\
&\left.U_{\left[\boldsymbol{s}_{k}^{\left.\boldsymbol{a},(p, n)_{]}\right]}\right.}\left(\boldsymbol{s}_{k}\right) \prod_{\ell=1}^{u(p)} \lambda_{T,\left(\boldsymbol{b}_{n}\right)_{\ell}} p\left(\boldsymbol{z}_{k}^{(\boldsymbol{a})_{\ell}} \mid\left[\boldsymbol{x}_{\left(\boldsymbol{b}_{n}\right)_{\ell}, k}^{(p)}\right]\right)\right), \quad(24)
\end{aligned}
$$

where $u^{(p)}$ is the number of consistent intervals which results in a non-empty relaxed intersection. In order to determine the contracted state, $\left[\boldsymbol{s}_{k}^{\boldsymbol{a},(p, n)}\right]$, the sub-states of each object are considered individually. The index for all the measurements assigned to object $i$, according to clustering, is defined by the set $\mathcal{B}$. Since only these measurements contract the substate of object $i$, the resulting contraction result for all measurements is given by $\left[\boldsymbol{x}_{i, k}^{\boldsymbol{a},(p, n)}\right]=\stackrel{\left\{|\mathcal{B}|-u_{i}^{(p)}\right\}}{\bigcap_{\ell \in \mathcal{B}}\left[\boldsymbol{x}_{i, k}^{\ell,(p, n)}\right] \text { with }}$ $u^{(p)}=\sum_{i \in \mathcal{I}^{(p)}} u_{i}^{(p)}$.

Considering the same example of 3 objects and 15 measurements, if the clustering algorithm results in 3 clusters, each indexing 5 of the measurements, the number of weighted box particles after the update per predicted box particle is reduced from 830584 with the approximate posterior in equation (23) to 27 with the approximate posterior in equation (24).

Two issues remain with the calculation of the approximate pdf in equation (24). Firstly, the relaxed intersection does not explicitly indicate the indices of the $u$ measurements which result in the non-zero intersection, which means it is not possible to evaluate the corresponding measurement likelihood for a single object expressions. Secondly, it is required to ensure that the box particle weight is represented by a single scalar value. If the measurement likelihood for a single object could be evaluated, this result may not be the case. There are several approaches which could be used to overcome this, such as selecting the midpoint of the box particle for measurement likelihood for a single object evaluation. However, the approach adopted in this paper overcomes both the remaining issues by approximating the measurement likelihood for a single object with a uniform distribution, as done previously in [1],

$$
p\left(\boldsymbol{z}_{k}^{j} \mid \boldsymbol{x}_{i, k}\right) \approx U_{r\left(\boldsymbol{x}_{i, k}\right)}\left(\boldsymbol{z}_{k}^{j}\right) .
$$

where $r\left(\boldsymbol{x}_{i, k}\right)$ represents the region in the measurement space where measurements from object $i$ may exist, based on the model of object $i$, and sensor noise characteristics. This approximation is valid if the uncertainty in the sensor error is significantly smaller than the extent of the object. In summary, the posterior at the previous time step, $p\left(\boldsymbol{s}_{k-1} \mid \boldsymbol{Z}_{1: k-1}\right)$, is approximated by $\left\{w_{k-1}^{(p)},\left[\boldsymbol{s}_{k-1}^{(p)}\right]\right\}_{p=1}^{N}$, and the posterior at the current time step, $p\left(\boldsymbol{s}_{k} \mid \boldsymbol{Z}_{1: k}\right)$ is approximated by $\left\{\left\{w_{k}^{(p, n)},\left[\boldsymbol{s}_{k}^{(p, n)}\right]\right\}_{p=1}^{N}\right\}_{n=1}^{\left|\mathcal{I}^{(p)}\right|^{d}}$, where $\left[\boldsymbol{s}_{k}^{(p, n)}\right]=\left[\boldsymbol{s}_{k}^{\boldsymbol{a},(p, n)}\right]$, and

$$
\begin{aligned}
& w_{k}^{(p, n)}=\frac{w_{k-1}^{(p)} e^{-\sum_{i \in \mathcal{I}}(p) \lambda_{T, i}}\left|\left[\boldsymbol{s}_{k}^{\boldsymbol{a},(p, n)}\right]\right| \rho^{M_{k}-u^{(p)}}}{\left|\left[\boldsymbol{s}_{k \mid k-1}^{(p)}\right]\right|} \times \\
& \prod_{\ell \in \mathcal{I}^{(p)}}\left(\frac{\lambda_{T, \ell}}{\left|\left[r\left(\boldsymbol{x}_{\ell, k}\right)\right]\right|}\right)^{u_{\ell}}
\end{aligned}
$$

\section{Box Particle Filter Resampling}

The number of box particles representing the posterior state pdf grows randomly with each time step. To curb the increase in the number of box particles, a resampling step is introduced, where the number of resampled particles is equal to the original number of box particles. In addition, the resampling step also relieves particle degeneracy. The resampling step in the Box PF differs from the resampling step of the generic PF. The resampling step in the Box PF can be performed by a division of box particles [32] (the box particle which has been selected $n$ times during resampling can be partitioned into $n$ disjoint smaller boxes) or by other techniques.

A summary of the Box PF for multiple extended object tracking is given in Algorithm I.

\section{Algorithm I. A Box Particle Filter for Multiple Extended Object Tracking}

Initialization

Initialize the box particles based on the available prior information about the objects kinematic and parameter state.

Repeat for $K$ time steps, $k=1, \ldots K$, the following steps:

1) Prediction

Propagate the box particles through the state evolution model to obtain the predicted box particles. Apply interval inclusion functions as described in [35]. (The implementation presented in this paper is based on the INTLAB [36] toolbox, which contains a number of built-in routines for interval calculations.)

\section{2) Measurement Update}

a) Upon receiving the measurements, form intervals around them, taking into account the uncertainty of the sensor, thus obtaining the measurement likelihood boxes $\left[\boldsymbol{Z}_{k}\right]$.

b) Cluster the measurements to obtain the set $\mathcal{C}_{i}$, where $i \in$ $1, \ldots, N_{c}$.

c) Solve the CSP in Eq. (19) using the CP algorithm to obtain the contracted box particles for each measurement $\left[\boldsymbol{s}_{k}^{a_{\ell},(p, n)}\right]$.

d) Determine the combined contracted box particle, $\left[\boldsymbol{s}_{k}^{\boldsymbol{a},(p, n)}\right]$, and the number of consistent intervals, $u$, through the calculation of the relaxed intersection.

e) Generate the set of weighted box particles according to equation (26).

3) Output

Obtain a box estimate for the state of the extended objects based on the maximum box particle weight:

$$
\left[\hat{\boldsymbol{x}}_{k}\right]=\arg \max _{\boldsymbol{x}_{k}} w_{k}^{(p)}
$$

and a point estimate $\hat{\boldsymbol{x}}_{k}$ for the extended shape using the midpoints of the box estimates of the state vector $\left[\hat{\boldsymbol{x}}_{k}\right]$.

4) Resampling

Resample $N$ particles with high weights by division. Finally, reset the weights: $w_{k}^{(p)}=1 / N$.

\section{PERformance Evaluation}

\section{A. Testing Environment}

The method performance is evaluated using data obtained from the HAMLeT (Hazardous Material Localization and 


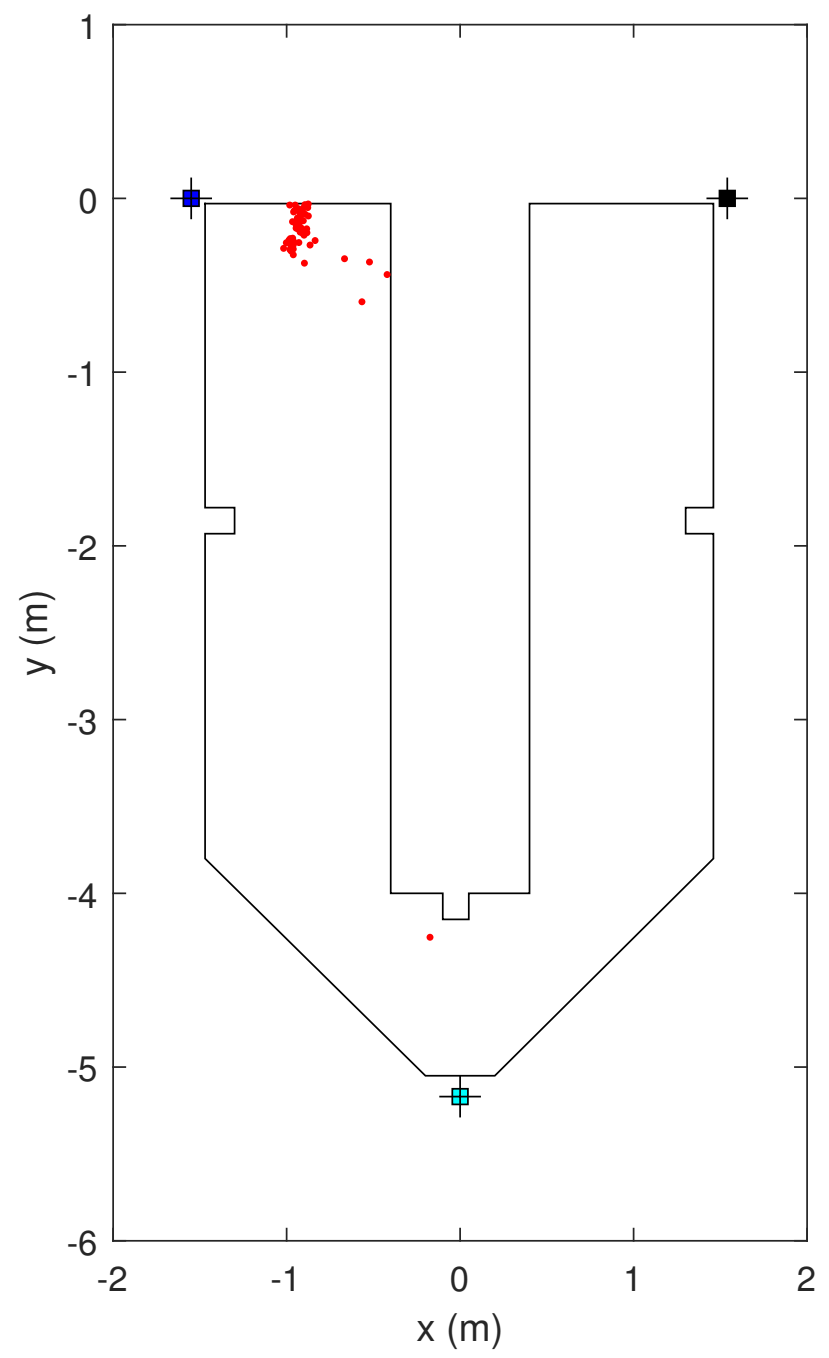

Fig. 2. The layout of the corridor for the experiments. The three laser scanner devices are indicated with crossed boxes. In this snapshot several measurements from the sensor located at the top left of the figure are displayed in red.

Person Tracking) system environment [37] (Fraunhofer FKIE, Germany). The data is from a prototype security system developed by an EU funded project, representing an airport corridor. This data consists of range and bearing components obtained by three laser rangefinder devices. The measurement devices are positioned at three key locations in a curved corridor (see Fig.2). The scenario presented in this paper consists of three people who enter and traverse the corridor while being observed by the sensors. The trajectories of the people is illustrated in Fig. 3.

Throughout their motion, each person moves in and out of the area visible by the sensors at different times. The sensors are positioned on the wall at the level of height of the hip.

\section{B. Person Modeling}

In the HAMLeT test environment it is of interest to track multiple people. Each person is modelled as an extended object in a two dimensional plane with a circular extent, as in Fig. 4. The sub-states of the state vector corresponding to the kinematics of each extended object is a vector,

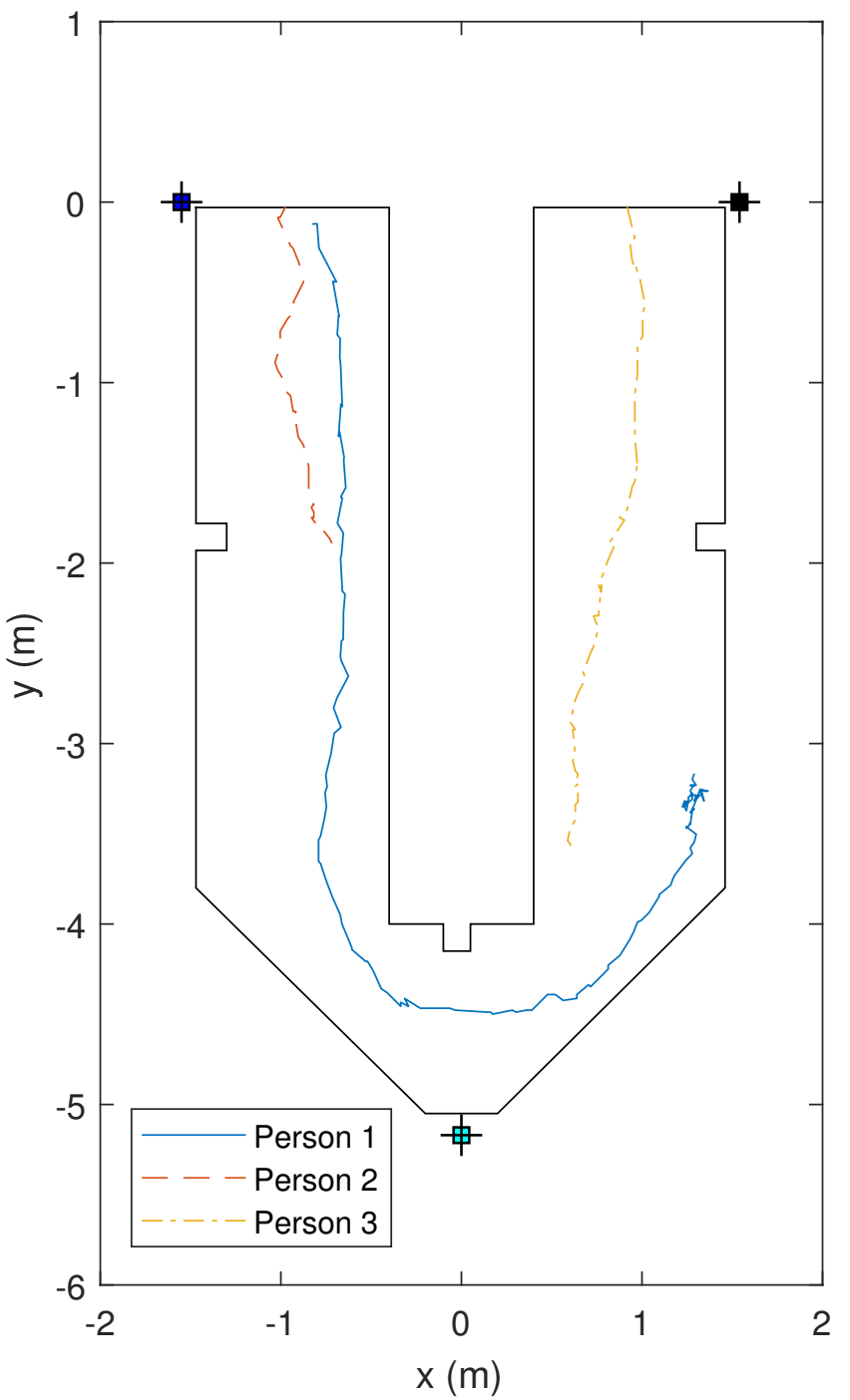

Fig. 3. Trajectories of the people moving through the corridor for the experiment.

$\boldsymbol{X}_{i, k}=\left(x_{i, k}^{c}, \dot{x}_{i, k}^{c}, y_{i, k}^{c}, \dot{y}_{i, k}^{c}\right)^{\top}$, which comprises of the position coordinates, $x_{i, k}^{c}, y_{i, k}^{c}$, and respective velocity components, $\dot{x}_{i, k}^{c}, \dot{y}_{i, k}^{c}$. In this scenario the subset of states of the state vector that comprises the parameters used to model the extent of each object reduces to a scalar representing the radius of the circle used to approximate the extent, $\Theta_{i, k}=R_{i, k}$.

In this paper the nearly constant velocity motion model [38] is considered as a representation of the motion of each person. In two dimensions, the motion of each extended object is thus given by

$$
\boldsymbol{X}_{i, k}=\boldsymbol{A} \boldsymbol{X}_{i, k-1}+\boldsymbol{\Gamma} \boldsymbol{\eta}_{X}
$$

where $\boldsymbol{A}=\operatorname{diag}\left(\boldsymbol{A}_{1}, \boldsymbol{A}_{1}\right), \boldsymbol{A}_{1}=\left(\begin{array}{cc}1 & T_{s} \\ 0 & 1\end{array}\right), \boldsymbol{\Gamma}=$ $\left(\begin{array}{cccc}T_{s}^{2} / 2 & T_{s} & 0 & 0 \\ 0 & 0 & T_{s}^{2} / 2 & T_{s}\end{array}\right)^{\top}, T_{s}$ is the sampling interval and $\boldsymbol{\eta}_{X} \sim \mathcal{N}\left(0, \boldsymbol{Q}_{X}\right)$ is the system dynamics noise, with covariance matrix $\boldsymbol{Q}_{X}$. It is assumed that $\boldsymbol{Q}_{X}=\operatorname{diag}\left(\boldsymbol{Q}_{1} \sigma_{x}^{2}, \boldsymbol{Q}_{1} \sigma_{y}^{2}\right)$, where $\boldsymbol{Q}_{1}=\left(\begin{array}{cc}T_{s}^{4} / 4 & T_{s}^{3} / 2 \\ T_{s}^{3} / 2 & T_{s}^{2}\end{array}\right)$ and $\sigma_{x}$ and $\sigma_{y}$ are the acceleration noise standard deviations for the $x$ and $y$ coordinate, 


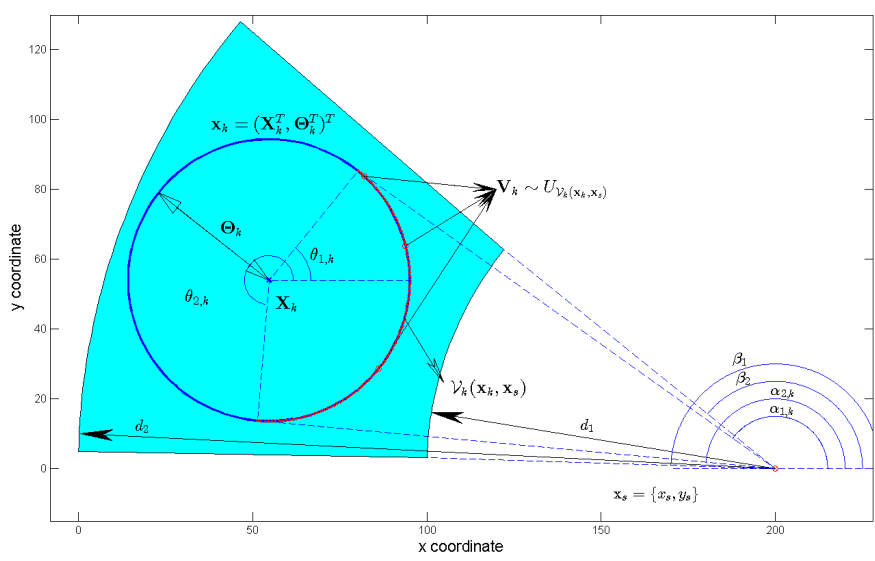

Fig. 4. Notations and definitions for a single circular extended object.

respectively. The evolution model for the extent parameter is assumed to be

$$
\Theta_{i, k}=\Theta_{i, k-1}+\eta_{\Theta}
$$

where $\eta_{\Theta} \sim \mathcal{N}\left(0, \sigma_{R}^{2}\right)$.

The visible border for a circular extended object is (see Fig. 4),

$$
\mathcal{V}_{k}\left(\boldsymbol{x}_{i, k}, \boldsymbol{x}_{s, k}\right)=\left(x_{i, k}^{c}+R_{i, k} \cos \left(\theta_{k}\right), y_{i, k}^{c}+R_{i, k} \sin \left(\theta_{k}\right)\right)
$$

where $\theta_{k} \in\left[\theta_{1, k}, \theta_{2, k}\right]$, with the $j$ th point source defined as,

$$
\boldsymbol{V}_{i, k}^{j}=\left(x_{i, k}^{c}+R_{i, k} \cos \left(\theta_{k}^{j}\right), y_{i, k}^{c}+R_{i, k} \sin \left(\theta_{k}^{j}\right)\right) .
$$

The measurement $\boldsymbol{z}_{k}^{j}$ collected from a sensor is in polar coordinates and consists of range $d_{k}^{j}$ and bearing $\beta_{k}^{j}$. The observation equation is thus given by

$$
\boldsymbol{z}_{k}^{j}=\left(d_{k}^{j}, \beta_{k}^{j}\right)^{\top}=h\left(\boldsymbol{V}_{i, k}^{j}\right)+\boldsymbol{w}_{k}^{j},
$$

where $h(\cdot)$ is

$$
\begin{aligned}
& h\left(\boldsymbol{V}_{i, k}^{j}\right)= \\
& \left(\begin{array}{c}
\sqrt{\left(x_{i, k}^{c}+R_{i, k} \cos \left(\theta_{k}^{j}\right)-x_{s, k}\right)^{2}+\left(y_{i, k}^{c}+R_{i, k} \sin \left(\theta_{k}^{j}\right)-y_{s, k}\right)^{2}} \\
\tan ^{-1}\left(\frac{y_{i, k}^{c}+R_{i, k} \sin \left(\theta_{k}^{j}\right)-y_{s, k}}{x_{i, k}^{c}+R_{i, k} \cos \left(\theta_{k}^{j}\right)-x_{s, k}}\right)
\end{array}\right) .
\end{aligned}
$$

The measurement noise $\boldsymbol{w}_{k}^{j}=\left(w_{d, k}^{j}, w_{\beta, k}^{j}\right)^{\top}$, is zero mean Gaussian, with a known covariance matrix $\Sigma=\operatorname{diag}\left(\sigma_{d}^{2}, \sigma_{\beta}^{2}\right)$.

The application of the $\mathrm{CP}$ for a circular extended object in the Box PF is summarized in Algorithm II and illustrated in Fig. 5.

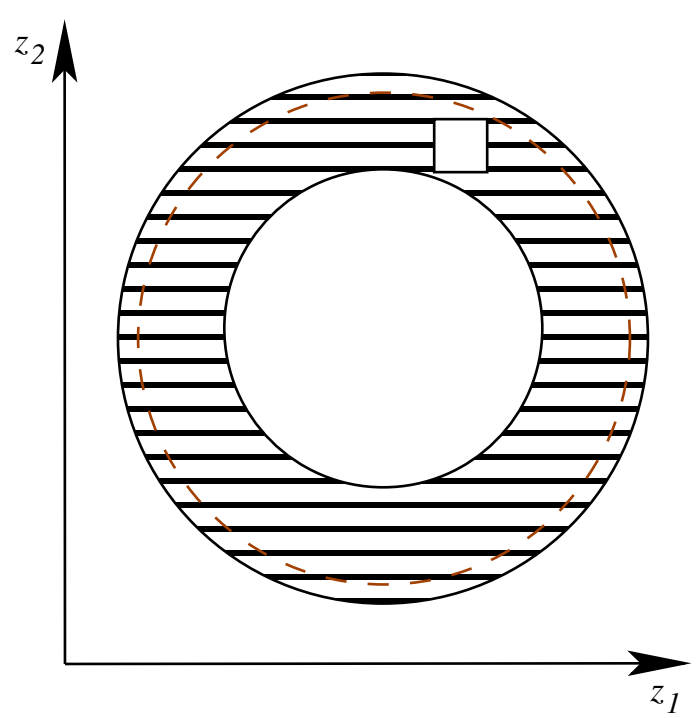

Fig. 5. Illustration of the contraction of a box particle by a single measurement for a circular extended object. The square box represents a measurement. The filled circular region represents the projection of a box particle sub-states for a single object to the measurement space. The dotted line illustrates the reduction in the interval shape due to contraction by the measurement.

\section{Algorithm II. Solving the CSP For Circular Extended Object Tracking}

1) Transform the range and bearing measurements into the $x-y$ plane using an inclusion function:

$$
\begin{aligned}
& {\left[z_{1}^{j}\right]=\left[C_{z_{1}}\right]\left(\left[d_{k}^{j}\right],\left[\beta_{k}^{j}\right]\right),} \\
& {\left[z_{2}^{j}\right]=\left[C_{z_{2}}\right]\left(\left[d_{k}^{j}\right],\left[\beta_{k}^{j}\right]\right),}
\end{aligned}
$$

where $\left[z_{1}^{j}\right]$ and $\left[z_{2}^{j}\right]$ represent the $x$ and $y$ dimension converted box measurements respectively, and $\left[C_{z_{1}}\right](\cdot)$ and $\left[C_{z_{2}}\right](\cdot)$ represent the corresponding inclusion functions.

2) Perform contraction on the sub-states corresponding to each object for each particle and measurement based on the following equations

$$
\begin{aligned}
& {\left[R_{k}^{(p)}\right]=\left[R_{k}^{(p)}\right] \cap\left(\sqrt{\left(\left[z_{1}^{j}\right]-\left[x_{c, k}^{(p)}\right]\right)^{2}+\left(\left[z_{2}^{j}\right]-\left[y_{c, k}^{(p)}\right]\right)^{2}}\right),} \\
& {\left[x_{c, k}^{(p)}\right]=\left[x_{c, k}^{(p)}\right] \cap\left(\left[z_{1}^{j}\right] \pm \sqrt{\left[R_{k}^{(p)}\right]^{2}-\left(\left[z_{2}^{j}\right]-\left[y_{c, k}^{(p)}\right]\right)^{2}}\right),} \\
& {\left[\dot{x}_{c, k}^{(p)}\right]=\left[\dot{x}_{c, k}^{(p)}\right] \cap\left(\frac{\left[x_{c, k}^{(p)}\right]-\left[x_{c, k-1}^{(p)}\right]}{T_{s}}\right),} \\
& {\left[y_{c, k}^{(p)}\right]=\left[y_{c, k}^{(p)}\right] \cap\left(\left[z_{2}^{j}\right] \pm \sqrt{\left[R_{k}^{(p)}\right]^{2}-\left(\left[z_{1}^{j}\right]-\left[x_{c, k}^{(p)}\right]\right)^{2}}\right),} \\
& {\left[\dot{y}_{c, k}^{(p)}\right]=\left[\dot{y}_{c, k}^{(p)}\right] \cap\left(\frac{\left[y_{c, k}^{(p)}\right]-\left[y_{c, k-1}^{(p)}\right]}{T_{s}}\right),} \\
& {\left[z_{1, j}^{(p)}\right]=\left[z_{1}^{j}\right] \cap\left(\left[x_{c, k}^{(p)}\right] \pm \sqrt{\left[R_{k}^{(p)}\right]^{2}-\left(\left[z_{2}^{j}\right]-\left[y_{c, k}^{(p)}\right]\right)^{2}}\right),} \\
& {\left[z_{2, j}^{(p)}\right]=\left[z_{2}^{j}\right] \cap\left(\left[x_{c, k}^{(p)}\right] \pm \sqrt{\left[R_{k}^{(p)}\right]^{2}-\left(\left[z_{1, j}^{(p)}\right]-\left[x_{c, k}^{(p)}\right]\right)^{2}}\right) .}
\end{aligned}
$$

3) Contract the original measurements with the contracted converted measurements:

$$
\begin{aligned}
& {\left[d_{k}^{(p), j}\right]=\left[d_{k}^{j}\right] \cap\left[C_{z_{1}}^{-1}\right]\left(\left[z_{1, j}^{(p)}\right],\left[z_{2, j}^{(p)}\right]\right),} \\
& {\left[\beta_{k}^{(p), j}\right]=\left[\beta_{k}^{j}\right] \cap\left[C_{z_{2}}^{-1}\right]\left(\left[z_{1, j}^{(p)}\right],\left[z_{2, j}^{(p)}\right]\right),}
\end{aligned}
$$

4) Repeat steps 2) and 3) sequentially until the gain in amount of contraction is sufficiently small. The amount of contraction could be checked or the steps could be iterated a fixed amount of times. 


\section{Performance Comparison}

The algorithms' evaluation is performed on a mobile computer with Intel(R) Core(TM) i7-4702HQ CPU @ 2.20GHz and with 16 GB of RAM. A comparison is made between the performance of the Box PF, a state-of-the-art PF, here after referred to as the border parameterized (BP) $\mathrm{PF}$, and the output of a purely clustering approach. The BP PF is the extended object tracking PF presented in [30] adapted for a varying number of extended objects, further details can be found in Appendix B. The clustering approach used is the DBSCAN algorithm [39]. This is a density based clustering algorithm which groups the measurements that are closely packed together into a single cluster. This clustering algorithm is well suited to the problem as it does not require knowledge of the number of clusters, and the density of the measurements from each object is consistent. The centroid of an object is estimated as the mean of all measurements assigned as a cluster. The radius of an object is approximated as the maximal distance between the centroid and any measurement assigned to the cluster. A threshold variable was introduced to reduce the effects of clutter. The results are averaged over a total of 100 independent runs where the measurements are perturbed by the measurement noise for each run. The performance is evaluated based on the Optimal Sub-Pattern Assignment (OSPA) [40] for the position of the objects, cardinality for the existent variables, the statistics of the existent object extents, and the average simulation time.

\section{Filter Parameters and initialization}

The Box PF utilizes the output of the DBSCAN algorithm. DBSCAN requires two parameters, $\epsilon=0.43$, related to the density of the clusters, and the minimum number of points required to form a dense region, which is selected as 1 . In this simulation the region $r\left(\boldsymbol{x}_{i, k}\right)$ is represented by equation (31).

The other parameters used in simulation for the performance evaluation are as follow: $\sigma_{x}=0.05 \mathrm{~m} / \mathrm{s}^{2}, \sigma_{y}=0.05 \mathrm{~m} / \mathrm{s}^{2}$, $\sigma_{R}=0.05 \mathrm{~m}, \sigma_{d}=0.025 \mathrm{~m}, \sigma_{\beta}=0.1 \pi / 180 \mathrm{rad}, T_{s}=1 \mathrm{~s}$, $\lambda_{T}=50, \rho=1 \times 10^{-4}, P_{e}=0.9, F=30$.

The filters utilize a uniform distribution to initialize each object sub-state when an object birth occurs. In the case of the Box PF, the same uniform region where the BP PF randomly generate particles from is subdivided so that the entire region is encompassed by all the box particles. This region, for each object sub-state, is located at the entrance/exit of the corridor: $\left[x_{c}\right]=[-1.5,-0.5] \cup[0.5,1.5] \mathrm{m},\left[\dot{x}_{c}\right]=[-0.1,0.1] \mathrm{m} / \mathrm{s}$, $\left[y_{c}\right]=[-1,0] \mathrm{m},\left[\dot{y}_{c}\right]=[-0.1,0.1] \mathrm{m} / \mathrm{s},[R]=[0,0.3] \mathrm{m}$.

\section{E. Results}

The performance of the filters is examined for 3 cases: a small, medium, and large number of particles. The average OSPA results for each case are illustrated in Fig. 6 to 8 . The spikes in the results correspond to a mismatch in cardinality. In the case of the Box PF and BP PF, this is caused by the fact that only a small number of measurements are observed from the objects when they first enter the observable region of a sensor. In the case of the clustering algorithm, the spikes

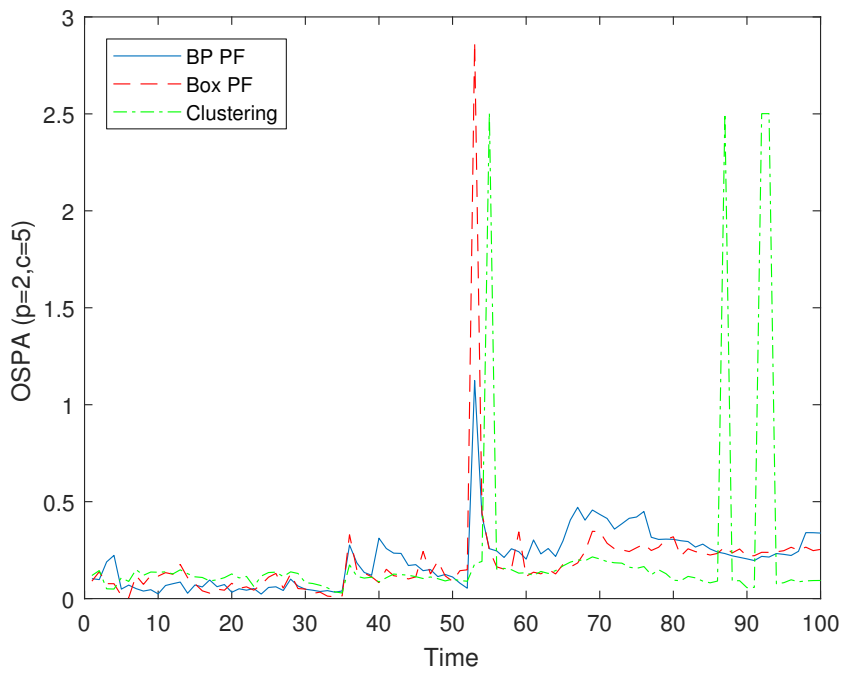

Fig. 6. Comparison of the average OSPA for the BP PF with 5000 particles, the Box PF with 32 particles, and DBSCAN clustering.

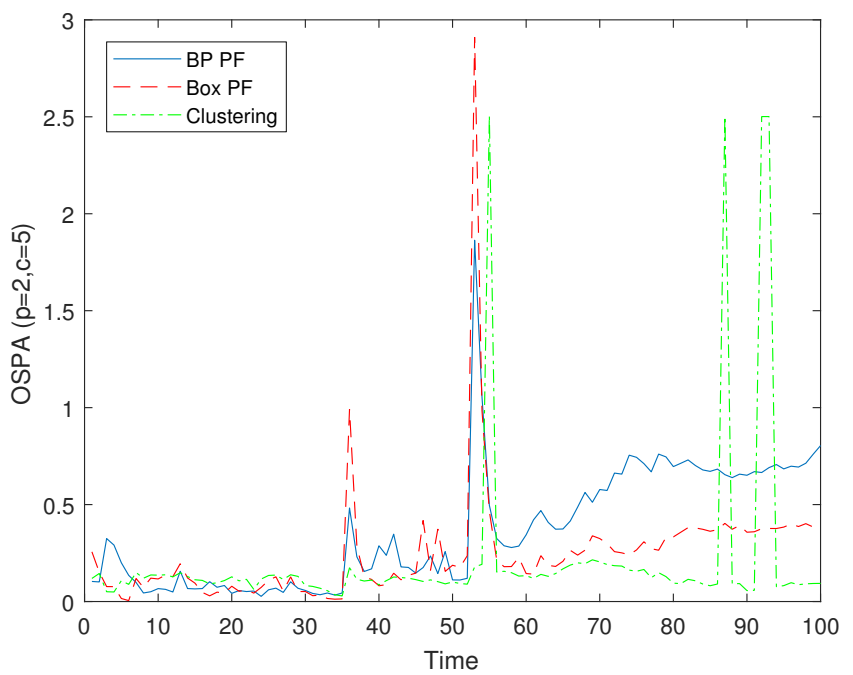

Fig. 7. Comparison of the average OSPA for the BP PF with 2500 particles, the Box PF with 16 particles, and DBSCAN clustering.

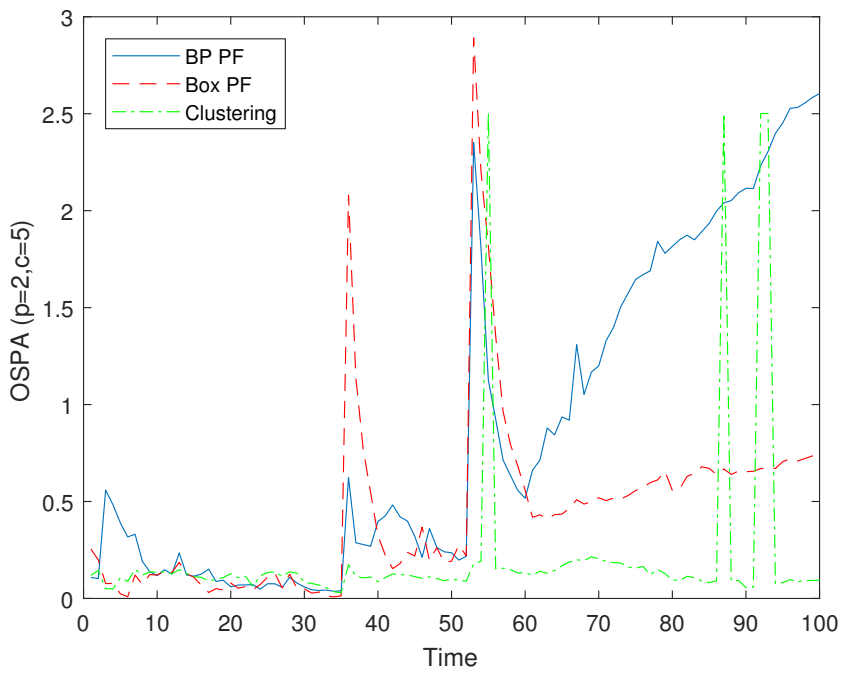

Fig. 8. Comparison of the average OSPA for the BP PF with 1000 particles, the Box PF with 4 particles, and DBSCAN clustering. 


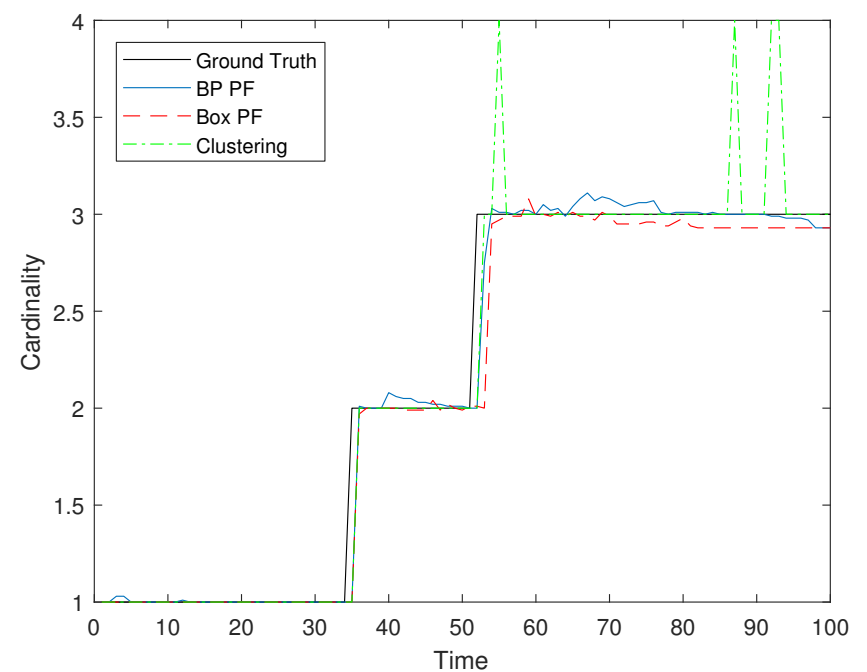

Fig. 9. Comparison of the average cardinality for the BP PF with 5000 particles, the Box PF with 32 particles, and DBSCAN clustering.

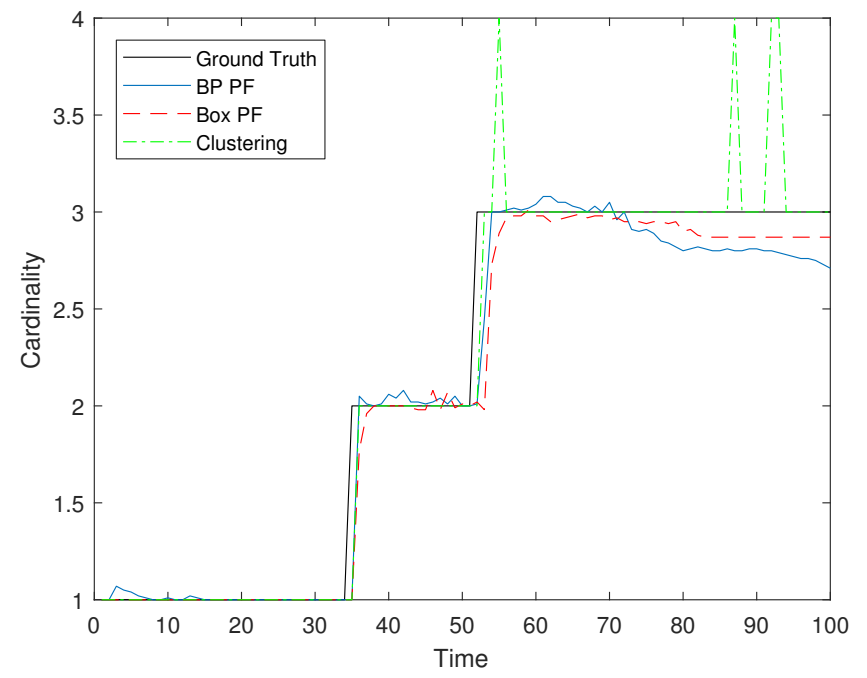

Fig. 10. Comparison of the average cardinality for the BP PF with 2500 particles, the Box PF with 16 particles, and DBSCAN clustering.

are caused by poorly selected parameters in the clustering algorithm. This highlights the robustness of the Box PF to the selection of parameters for the clustering algorithm. When cardinality errors are not present, the clustering algorithm performs on par with the filtering approaches with respect to OSPA. This is aligned with the findings in [41], [42] where clustering algorithms are applied in the context of object tracking.

In the case of the filters, decreasing the number of particles increases the amount of error, however, it is worth noting that a decrease in the number of particles for the BP PF also causes the filter to become unstable when three objects are within the scene.

The average cardinality results for each case are illustrated in Fig. 9 to 11. The cardinality of the Box PF is significantly more robust to different numbers of box particles and clustering parameters.

The performance of the approaches for the estimation of

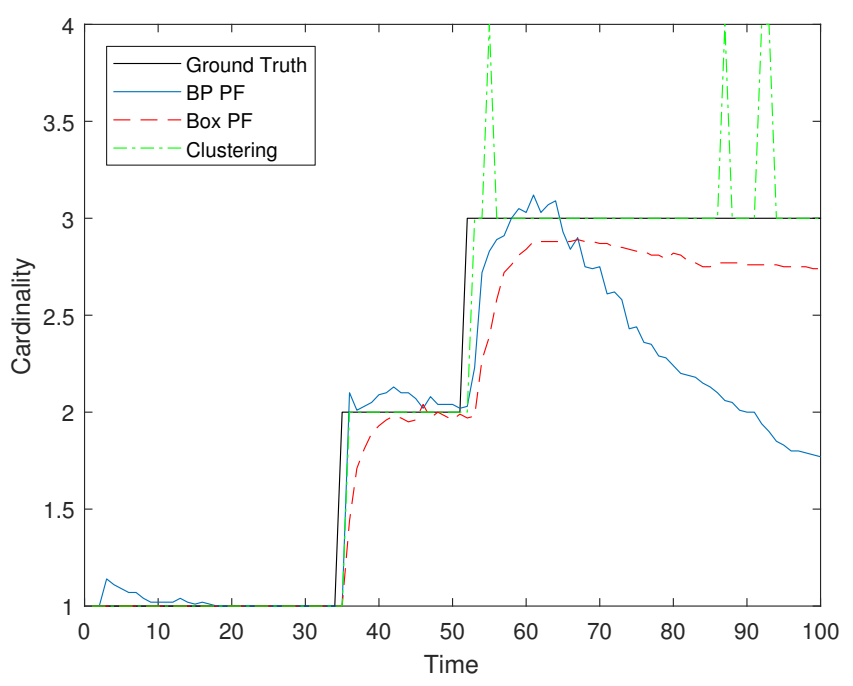

Fig. 11. Comparison of the average cardinality for the BP PF with 1000 particles, the Box PF with 4 particles, and DBSCAN clustering.

TABLE I

EXISTENT OBJECT EXTENT STATISTICS.

\begin{tabular}{|l|l|c|c|}
\hline Algorithm & $N$ & Mean $(\mathrm{m})$ & $\begin{array}{l}\text { Standard } \\
\text { Deviation }(\mathrm{m})\end{array}$ \\
\hline \multirow{3}{*}{ Box PF } & 4 & 0.23 & 0.03 \\
\cline { 2 - 4 } & 16 & 0.21 & 0.04 \\
\cline { 2 - 4 } & 32 & 0.2 & 0.05 \\
\hline \multirow{3}{*}{ BP PF } & 1000 & 0.24 & 0.13 \\
\cline { 2 - 4 } & 2500 & 0.23 & 0.13 \\
\cline { 2 - 4 } & 5000 & 0.21 & 0.11 \\
\hline Clustering & & 0.3 & 0.07 \\
\hline
\end{tabular}

the extent parameter is illustrated by the mean and standard deviation of the extent parameter for all active objects over all time steps, this is illustrated in Table I. The contraction operation leads to the Box PF sustaining significantly lower variations in the extent, even with higher numbers of particles. The rudimentary approach of approximating the extent in the clustering approach leads to an over estimate of the extent which is sensitive to clutter.

The computational time for each of the considered cases under the same conditions is given in Table II. It is noted here that employing the INTLAB [36] toolbox with MATLAB for performing the Box PF simulation is just one way of implementing the Box PF code. This toolbox was initially designed and optimized for estimating rounding errors. Faster realizations of the Box $\mathrm{PF}$ in $\mathrm{C} / \mathrm{C}++$ are also possible. For in-

TABLE II

AVERAgE Matlab COMPUTATIONAL TIME COMPARISON.

\begin{tabular}{|l|l|c|}
\hline Algorithm & $N$ & Computation Time (s) \\
\hline \multirow{3}{*}{ Box PF } & 4 & 43.38 \\
\cline { 2 - 3 } & 16 & 118.56 \\
\cline { 2 - 3 } & 32 & 282.24 \\
\hline \multirow{3}{*}{ BP PF } & 1000 & 67.68 \\
\cline { 2 - 3 } & 2500 & 168.53 \\
\cline { 2 - 3 } & 5000 & 417.66 \\
\hline Clustering & & 4.25 \\
\hline
\end{tabular}


stance, in [43] the Box Probability Hypothesis Density Filter is shown to be 10.9 times faster than the Probability Hypothesis Density Filter working with point particles (both implemented in $\mathrm{C}++$ ). Further optimization is considered possible for the Box PF realization, thus the results in Table II would represent a minimum efficiency improvement. The Box PF is also a very attractive solution from the perspective of distributed estimation, as shown in [44]. The clustering approach offers the greatest computational saving, but this comes at a cost of poor estimation of the objects extents which are sensitive to clutter.

An attractive benefit of the Box PF, not clearly illustrated in the results presented thus far, is the ability of the filter to handle large regions of initial uncertainty. For example, the prior distribution on the sub-states related to the velocity components of each object is a uniform distribution with the following region of support: $[-0.1,0.1]$. This region caters for objects moving in any direction and was sufficient for the objects in the examined scenario, but when the magnitude is increased, the BP PF is unable to lock on to new born objects. This is due to the fact that the velocity of the object is not directly observed, causing the filter to diverge. However, due to contraction and the division of boxes in the resampling step, the Box PF is capable of handling larger regions of uncertainty. As an example, increasing the region to $[-1,1]$, caused the BP PF to diverge in all three cases, where the Box $\mathrm{PF}$ performance was unaffected. This issue can be resolved by the BP PF by utilizing a larger number of particles, but this comes at the cost of an even greater computational complexity. In addition, filtering approaches have track continuity when the detection probability is low. This enables the estimates (by using the priors) to be more robust to misdetection. In scenarios with inaccurate sensors, it is also anticipated that filtering approaches would benefit from the evolution model to maintain tracking.

\section{CONCLUSIONS}

This paper presents a Box PF method for multiple extended object tracking. A theoretical derivation of the generalized likelihood function of the Box PF in the presence of clutter is presented. The proved equation is further modified to minimize the computational complexity. It is shown that the Box PF can work efficiently with four to thirty two box particles, whereas the particle filter working with point particles needs hundreds and thousands of particles to achieve the same accuracy. The Box PF has been shown to have several advantages when compared to the BP PF. This includes a significant computational gain, more than $32 \%$, which could potentially be further exploited through an implementation on a platform that is efficient in interval arithmetic. The Box PF exhibits robustness for a significantly smaller number of box particles which completely encompass the initialization region. The performance of the method is validated with real data from laser rangefinder sensors.

Future work will focus on the expansion and validation of the method for more general application settings, such as asynchronous multirate sensor networks [45], and correlated sensor noise [46].
Acknowledgments. We acknowledge the support from the UK Engineering and Physical Sciences Research Council (EPSRC) for the support via the Bayesian Tracking and Reasoning over Time (BTaRoT) grant EP/K021516/1 and EC Seventh Framework Programmes [FP7 2007-2013] Monte Carlo based Innovative Management and Processing for an Unrivalled Leap in Sensor Exploitation (MC-Impulse) Grant agreement no.: 238710, and [FP7 2013-2017] TRAcking in compleX sensor systems (TRAX) Grant agreement no.: 607400.

\section{APPENDIX}

\section{A. Expanded Generalized Likelihood Proof}

According to equation (15), given $\mathcal{I}$, for any value of $M_{k}$ :

$$
\begin{gathered}
\prod_{m=1}^{M_{k}}\left(\rho+\sum_{i \in \mathcal{I}} \lambda_{T, i} p\left(\boldsymbol{z}_{k}^{m} \mid \boldsymbol{x}_{i, k}\right)\right)=\rho^{M_{k}}+\sum_{m=1}^{M_{k}} \sum_{j=1}^{\left(\begin{array}{l}
M_{k} \\
m
\end{array}\right)} \sum_{n=1}^{|\mathcal{I}|^{m}} \rho^{M_{k}-m} \times \\
\prod_{\ell=1}^{m} \lambda_{T,\left(\boldsymbol{b}_{m, n}\right)_{\ell}} p\left(\boldsymbol{z}_{k}^{\left(\boldsymbol{a}_{m, j}\right)_{\ell}} \mid \boldsymbol{x}_{\left(\boldsymbol{b}_{m, n}\right)_{\ell}, k}\right) .
\end{gathered}
$$

To simplify notations, define: $c_{m, i}=p\left(\boldsymbol{z}_{k}^{m} \mid \boldsymbol{x}_{i, k}\right)$ and $\mathcal{C}\left(c_{1, \mathcal{I}}, c_{2, \mathcal{I}}, \ldots, c_{M_{k}, \mathcal{I}} ; \psi\right)$ represents the summation of all $\psi$ unique combinations of $c_{m, i}$ terms multiplied by the associated object densities, with $\mathcal{C}\left(c_{1, \mathcal{I}}, c_{2, \mathcal{I}}, \ldots, c_{M_{k}, \mathcal{I}} ; 0\right)=1, \mathcal{C}\left(c_{1, \mathcal{I}}, c_{2, \mathcal{I}}, \ldots, c_{M_{k}, \mathcal{I}} ;-1\right)=$ 0 , and $\mathcal{C}\left(c_{1, \mathcal{I}}, c_{2, \mathcal{I}}, \ldots, c_{M_{k}, \mathcal{I}} ; M_{k}+1\right)=0$. For example, if $\mathcal{I}=\{1,2\}$, then $\mathcal{C}\left(c_{1, \mathcal{I}}, c_{2, \mathcal{I}}, c_{3, \mathcal{I}} ; 2\right)=$ $\lambda_{T, 1}^{2} c_{1,1} c_{2,1}+\lambda_{T, 1} \lambda_{T, 2} c_{1,1} c_{2,2}+\lambda_{T, 1} \lambda_{T, 2} c_{1,2} c_{2,1}+$ $\lambda_{T, 2}^{2} c_{1,2} c_{2,2}+\lambda_{T, 1}^{2} c_{2,1} c_{3,1}+\lambda_{T, 1} \lambda_{T, 2} c_{2,1} c_{3,2}+$ $\lambda_{T, 1} \lambda_{T, 2} c_{2,2} c_{3,1}+\lambda_{T, 2}^{2} c_{2,2} c_{3,2}+\lambda_{T, 1}^{2} c_{1,1} c_{3,1}+$ $\lambda_{T, 1} \lambda_{T, 2} c_{1,1} c_{3,2}+\lambda_{T, 1} \lambda_{T, 2} c_{1,2} c_{3,1}+\lambda_{T, 2}^{2} c_{1,2} c_{3,2}$. $\quad \mathrm{A}$ useful decomposition of the expression in this form is: $\mathcal{C}\left(c_{1, \mathcal{I}}, c_{2, \mathcal{I}}, \ldots, c_{M_{k}+1, \mathcal{I}} ; \psi\right)=\mathcal{C}\left(c_{1, \mathcal{I}}, c_{2, \mathcal{I}}, \ldots, c_{M_{k}, \mathcal{I}} ; \psi\right)+$ $\sum_{i \in \mathcal{I}} \lambda_{T, i} c_{M_{k}+1, i} \mathcal{C}\left(c_{1, \mathcal{I}}, c_{2, \mathcal{I}}, \ldots, c_{M_{k}, \mathcal{I}} ; \psi-1\right)$. The compact form of equation (37) is

$$
\prod_{m=1}^{M_{k}}\left(\rho+\sum_{i \in \mathcal{I}} \lambda_{T, i} c_{m, i}\right)=\sum_{m=0}^{M_{k}} \rho^{M_{k}-m} \mathcal{C}\left(c_{1, \mathcal{I}}, c_{2, \mathcal{I}}, \ldots, c_{M_{k}, \mathcal{I}} ; m\right) .
$$

Base case: $M_{k}=1$ :

$$
\begin{aligned}
\rho+\sum_{i \in \mathcal{I}} \lambda_{T, i} c_{1, i} & =\sum_{m=0}^{1} \rho^{1-m} \mathcal{C}\left(c_{1, \mathcal{I}} ; m\right) \\
& =\rho^{1-0} \mathcal{C}\left(c_{1, \mathcal{I}} ; 0\right)+\rho^{1-1} \mathcal{C}\left(c_{1, \mathcal{I}} ; 1\right) \\
& =\rho+\sum_{i \in \mathcal{I}} \lambda_{T, i} c_{1, i} .
\end{aligned}
$$

Inductive hypothesis: Suppose equation (38) holds for all values of $M_{k}$.

Inductive step: Consider the scenario with $M_{k}+1$ measure- 
ments,

$$
\begin{aligned}
\prod_{m=1}^{M_{k}+1}(\rho & \left.+\sum_{i \in \mathcal{I}} \lambda_{T, i} c_{m, i}\right)= \\
& {\left[\prod_{m=1}^{M_{k}}\left(\rho+\sum_{i \in \mathcal{I}} \lambda_{T, i} c_{m, i}\right)\right]\left(\rho+\sum_{i \in \mathcal{I}} \lambda_{T, i} c_{M_{k}+1, i}\right) } \\
= & \sum_{m=0}^{M_{k}} \rho^{M_{k}-m} \mathcal{C}\left(c_{1, \mathcal{I}}, c_{2, \mathcal{I}}, \ldots, c_{M_{k}, \mathcal{I}} ; m\right) \times \\
= & \sum_{m=0}^{M_{k}+1} \rho^{M_{k}+1-m} \mathcal{C}\left(c_{1, \mathcal{I}}, c_{2, \mathcal{I}}, \ldots, c_{M_{k}, \mathcal{I}} ; m\right) \\
+ & \sum_{i \in \mathcal{I}} \lambda_{T, i} \lambda_{T, i} c_{M_{k}+1, i} \sum_{m=0}^{M_{k}+1} \rho^{M_{k}+1-m} \times \\
= & \sum_{m=0}^{M_{k}+1} \rho^{M_{k}+1-m}\left(\mathcal{C}\left(c_{1, \mathcal{I}}, c_{2, \mathcal{I}}, \ldots, c_{M_{k}, \mathcal{I}} ; m\right)\right. \\
+ & \left.\sum_{i \in \mathcal{I}} \lambda_{T, i} c_{M_{k}+1, i} \mathcal{C}\left(c_{1, \mathcal{I}}, c_{2, \mathcal{I}}, \ldots, c_{M_{k}, \mathcal{I}} ; m-1\right)\right) \\
= & \sum_{m=0}^{M_{k}+1} \rho^{M_{k}+1-m} \mathcal{C}\left(c_{1, \mathcal{I}}, c_{2, \mathcal{I}}, \ldots, c_{M_{k}+1, \mathcal{I}} ; m\right) .
\end{aligned}
$$

By the principle of mathematical induction, the proposition holds for all $M_{k} \in \mathbb{N}$.

\section{B. Border Parameterized Particle Filter}

In [30] a PF for tracking multiple extended objects is presented. However, in this case the number of extended objects is fixed and known. This algorithm has been adapted to consider a varying number of extended objects as in Section II-A. With respect to the notation developed in Section II-B, the BP PF represents the posterior state pdf with the following discrete approximation

$$
p\left(\boldsymbol{s}_{k} \mid \boldsymbol{Z}_{1: k}\right)=\sum_{p=1}^{N} w_{k}^{(p)} \delta\left(\boldsymbol{s}_{k}-\boldsymbol{s}_{k}^{(p)}\right),
$$

where $\delta(\cdot)$ is the Dirac delta function, and the weights, $\left\{w_{k}^{(p)}\right\}_{p=1}^{N}$, are normalized so that $\sum_{p} w_{k}^{(p)}=1$.

The transition pdf, described in (4), is used to modify the set of particles, represented by $\left\{\boldsymbol{s}_{k \mid k-1}^{(p)}\right\}_{p=1}^{N}$. The weight of each particle is re-evaluated based on the latest set of measurements and the generalized likelihood function of (6).

Since the measurement likelihood for a single object, $p\left(\boldsymbol{z}_{k}^{j} \mid \boldsymbol{x}_{i, k}\right)$, is analytically intractable, a Monte Carlo method is used to approximate it, as in [17] and [47]. For each particle existent object subspace, $\boldsymbol{x}_{i, k \mid k-1}^{(p)}$, the support of $p\left(\boldsymbol{V}_{i, k}^{j} \mid \boldsymbol{x}_{i, k \mid k-1}^{(p)}\right)$ is defined by a uniform distribution over the angular range $\left[\theta_{1, k}, \theta_{2, k}\right]$ of the visible border $\mathcal{V}_{k}\left(\boldsymbol{x}_{i, k \mid k-1}^{(p)}, \boldsymbol{x}_{s, k}\right)$ with respect to the object center. A sampled point source can be obtained by first sampling from:

$$
\left\{\theta_{k}^{(b, f)}\right\}_{b=1, f=1}^{N, F} \sim\left(\mathcal{U}_{\left[\theta_{1, k}, \theta_{2, k}\right]}\left(\theta_{k}\right)\right),
$$

followed by the substitution of $\left\{\theta_{k}^{(b, f)}\right\}_{b=1, f=1}^{N, F}$ into equation (31), resulting in a random set of samples denoted as
$\mathcal{J}_{k}=\left\{\boldsymbol{V}_{i, k}^{j,(b, f)}\right\}_{b=1, f=1}^{N, F}$, where $F$ is the number of samples from the object border. The Monte Carlo approximation for the measurement likelihood for a single object is then given by:

$$
\begin{aligned}
p\left(\boldsymbol{z}_{k}^{j} \mid \boldsymbol{x}_{i, k \mid k-1}^{(p)}\right) & =\int p\left(\boldsymbol{z}_{k}^{j} \mid \boldsymbol{V}_{i, k}^{j}\right) p\left(\boldsymbol{V}_{i, k}^{j} \mid \boldsymbol{x}_{i, k \mid k-1}^{(p)}\right) d \boldsymbol{V}_{i, k}^{j}, \\
& \approx \frac{1}{F} \sum_{\boldsymbol{V}_{i, k}^{j} \in \mathcal{J}_{k}} p\left(\boldsymbol{z}_{k}^{j} \mid \boldsymbol{V}_{i, k}^{j}\right) .
\end{aligned}
$$

The BP PF is summarized in Algorithm III.

Algorithm III. The Border Parameterized Particle Filter for Multiple Extended Object Tracking

\section{Initialization}

$k=0$, Initialize the set of particles based on the available prior information, $\left\{\boldsymbol{s}_{0}^{(p)} \sim p\left(\boldsymbol{s}_{0}\right)\right\}$. Set initial weights $\left\{w_{0}^{(p)}\right\}_{p=1}^{N}=\frac{1}{N}$.

Repeat the following steps for $T$ time steps, $k=1, \ldots T$ :

\section{1) Prediction}

Propagate the particles, $\left\{\boldsymbol{s}_{k-1}^{(p)}\right\}_{p=1}^{N}$ with the state transition pdf (equation (4)) to obtain the predicted particles, $\left\{\boldsymbol{s}_{k \mid k-1}^{(p)}\right\}_{p=1}^{N}$.

2) Measurement Update

On the receipt of a new measurement:

a) Evaluate the measurement likelihood for a single object, $p\left(\boldsymbol{z}_{k}^{j} \mid \boldsymbol{x}_{i, k \mid k-1}^{(p)}\right)$, according to (43) for all the measurements.

b) Calculate the weights $w_{k}^{(p)}, p=1, \ldots, N$ using terms from step 2-a) and equation (6).

3) Output

Calculate the estimated state vector $\hat{\boldsymbol{x}}_{k}$ based on the maximum particle weight:

$$
\hat{\boldsymbol{x}}_{k}=\arg \max _{\boldsymbol{x}_{k}} w_{k}^{(p)}
$$

\section{4) Resampling}

a) Compute the effective sample size

$$
N_{e f f}=\frac{1}{\sum_{p=1}^{N}\left(\hat{w}_{k}^{(p)}\right)^{2}}
$$

b) Selection

If $N_{\text {eff }} \leq N_{\text {thresh }}$ (with e.g. $N_{\text {thresh }}=2 N / 3$ ) resample the particles. Finally, reset the weights: $w_{k}^{(p)}=1 / N$.

\section{REFERENCES}

[1] A. D. Freitas, L. Mihaylova, A. Gning, D. Angelova, and V. Kadirkamanathan, "Autonomous Crowds Tracking with Box Particle Filtering and Convolution Particle Filtering," Automatica, vol. 69, pp. 380-394, 2016.

[2] M. Feldmann and W. Koch, "Road-map Assisted Convoy Track Maintenance using Random Matrices," in Proc. of 11th Int. Conf. on Information Fusion, June 2008, pp. 1-8.

[3] D. Angelova and L. Mihaylova, "Extended Object Tracking Using Monte Carlo Methods," IEEE Trans. on Signal Processing, vol. 56, no. 2, pp. 825-832, Feb 2008

[4] A. Carmi, F. Septier, and S. J. Godsill, "The Gaussian Mixture MCMC Particle Algorithm for Dynamic Cluster Tracking," Automatica, vol. 48, no. 10 , pp. $2454-2467,2012$

[5] K. Granström, C. Lundquist, and U. Orguner, "Tracking Rectangular and Elliptical Extended Targets using Laser Measurements," in Proc. of 14th Int. Conf. on Information Fusion, July 2011, pp. 1-8.

[6] F. Septier, S. K. Pang, A. Carmi, and S. Godsill, "On MCMC-Based Particle Methods for Bayesian Filtering: Application to Multitarget Tracking," in Proc. of the IEEE Int. Workshop on Computational Advances in Multi-Sensor Adaptive Processing, Dec. 2009, pp. 360363 
[7] L. Mihaylova, A. Carmi, F. Septier, A. Gning, S. Pang, and S. Godsill, "Overview of Bayesian Sequential Monte Carlo Methods for Group and Extended Object Tracking," Digital Signal Processing: A Review Journal, vol. 25, no. 1, pp. 1-16, 2014.

[8] K. Granström and M. Baum, "Extended Object Tracking: Introduction, Overview and Applications," 2016, arXiv preprint arXiv:1604.00970.

[9] R. P. S. Mahler, "PHD Filters for Nonstandard Targets, I: Extended Targets," Proc. of 12th Int. Conf. on Information Fusion, pp. 915-921, 2009.

[10] K. Granström and U. Orguner, "A PHD Filter for Tracking Multiple Extended Targets Using Random Matrices," IEEE Trans. on Signal Processing, vol. 60, no. 11, pp. 5657-5671, Nov 2012.

[11] C. Lundquist, K. Granström, and U. Orguner, "An Extended Target CPHD Filter and a Gamma Gaussian Inverse Wishart Implementation," IEEE Journal of Selected Topics in Signal Processing, vol. 7, no. 3, pp. 472-483, June 2013.

[12] M. Beard, S. Reuter, K. Granström, B. T. Vo, B. N. Vo, and A. Scheel, "Multiple Extended Target Tracking With Labeled Random Finite Sets," IEEE Transactions on Signal Processing, vol. 64, no. 7, pp. 1638-1653, April 2016.

[13] M. Baum and U. Hanebeck, "Shape Tracking of Extended Objects and Group Targets with Star-convex RHMs," in Proc. of 14th Int. Conf. on Information Fusion, 2011.

[14] M. Baum and U. D. Hanebeck, "Extended Object Tracking Based on Set-Theoretic and Stochastic Fusion," IEEE Trans. on Aerospace and Electronic Systems, vol. 48, no. 4, pp. 3103-3115, Oct. 2012.

[15] J. Lan and X. Li, "Tracking of Maneuvering Non-Ellipsoidal Extended Object or Target Group Using Random Matrix," IEEE Trans. on Signal Processing, vol. 62, no. 9, pp. 2450-2463, May 2014.

[16] K. Gilholm and D. Salmond, "Spatial Distribution Model for Tracking Extended Objects," IEE Proc. Radar, Sonar and Navigation, vol. 152, no. 5, pp. 364-371, Oct. 2005.

[17] Y. Boers, H. Driessen, J. Torstensson, M. Trieb, R. Karlsson, and F. Gustafsson, "Track-before-detect Algorithm for Tracking Extended Targets," IEE Proc. Radar, Sonar and Navigation, vol. 153, no. 4, pp. 345-351, Aug. 2006.

[18] J. Koch, "Bayesian Approach to Extended Object and Cluster Tracking using Random Matrices," IEEE Trans. on Aerospace and Electronic Systems, vol. 44, no. 3, pp. 1042-1059, July 2008.

[19] M. Baum and U. D. Hanebeck, "Extended Object Tracking with Random Hypersurface Models," IEEE Trans. on Aerospace and Electronic Systems, vol. 50, no. 1, pp. 149-159, Jan. 2014.

[20] N. Wahlström and E. Özkan, "Extended Target Tracking Using Gaussian Processes," IEEE Trans. on Signal Processing, vol. 63, no. 16, pp. 41654178, Aug. 2015.

[21] F. Abdallah, A. Gning, and P. Bonnifait, "Box Particle Filtering for Nonlinear State Estimation using Interval Analysis ", Automatica, vol. 44, no. 3, pp. 807 - 815, March 2008.

[22] N. Merlinge, J. Marzat, and L. Reboul, "Optimal guidance and observer design for target tracking using bearing-only measurements," ONERA, France, Tech. Rep., 2016

[23] N. Merlinge, K. Dahla, and H. Piet-Lahanier, "A Box Regularized Particle Filter for terrain navigation with highly non-linear measurements," in Proc. of the 20th IFAC Symposium on Automatic Control in Aerospace (ACA), vol. 49, no. 17, 2016, pp. 361-366.

[24] A. Gning, L. Mihaylova, and F. Abdallah, "Mixture of Uniform Probability Density Functions for Nonlinear State Estimation Using Interval Analysis," in Proc. of 13th Int. Conf. on Information Fusion, 2010, pp. $1-8$.

[25] A. Gning, L. Mihaylova, F. Abdallah, and B. Ristic, "Particle Filtering Combined with Interval Methods for Tracking Applications," in Integrated Tracking, Classification, and Sensor Management: Theory and Applications, M. Mallick, V. Krishnamurthy, and B.-N. Vo, Eds. John Wiley \& Sons, New Jersey, USA, 2012, pp. 43-74.

[26] N. Petrov, M. Ulmke, L. Mihaylova, A. Gning, M. Schikora, M. Wieneke, and W. Koch, "On the Performance of the Box Particle Filter for Extended Object Tracking Using Laser Data," in Workshop on Sensor Data Fusion, Sept. 2012, pp. 19 -24.
[27] N. Petrov, A. Gning, L. Mihaylova, and D. Angelova, "Box Particle Filtering for Extended Object Tracking," in Proc. of 15th Int. Conf. on Information Fusion, July 2012, pp. 82 - 89.

[28] J. Vermaak, N. Ikoma, and S. Godsill, "Sequential Monte Carlo Framework for Extended Object Tracking," IEE Proc. Radar, Sonar and Navigation, vol. 152, no. 5, pp. 353-363, Oct. 2005.

[29] F. Septier, J. Cornebise, S. Godsill, and Y. Delignon, "A Comparative Study of Monte-Carlo Methods for Multitarget Tracking," in IEEE Statistical Signal Processing Workshop, June 2011, pp. 205-208

[30] K. Gilholm, S. Godsill, S. Maskell, and D. Salmond, "Poisson Models for Extended Target and Group Tracking," in Optics \& Photonics 2005, vol. 5913. International Society for Optics and Photonics, 2005.

[31] A. Gning, B. Ristic, L. Mihaylova, and F. Abdallah, "An Introduction to Box Particle Filtering [Lecture Notes]," IEEE Signal Processing Magazine, vol. 30, no. 4, pp. 166-171, July 2013.

[32] A. Gning, B. Ristic, and L. Mihaylova, "Bernoulli Particle/Box-Particle Filters for Detection and Tracking in the Presence of Triple Measurement Uncertainty," IEEE Trans. on Signal Processing, vol. 60, no. 5, pp. 2138-2151, May 2012.

[33] L. Jaulin, M. Kieffer, O. Didrit, and E. Walter, Applied Interval Analysis. Springer-Verlag, 2001.

[34] L. Jaulin, "Robust Set-membership State Estimation; Application to Underwater Robotics," Automatica, vol. 45, no. 1, pp. 202-206, Jan. 2009.

[35] - ,Nonlinear Bounded-error State Estimation of Continuous-time Systems," Automatica, vol. 38, no. 6, pp. 1079 - 1082, June 2002.

[36] S. Rump, "INTLAB - INTerval LABoratory," in Developments in Reliable Computing, T. Csendes, Ed. Dordrecht: Kluwer Academic Publishers, 1999, pp. 77-104, http://www.ti3.tu-harburg.de/rump/.

[37] M. Wieneke, K. Safenreiter, and W. Koch, "Combined Person Tracking and Classification in a Network of Chemical Sensors," in Proc. of 11th Int. Conf. on Information Fusion, June 2008, pp. 1-8.

[38] Y. Bar-Shalom, X.-R. Li, and T. Kirubarajan, Estimation with Applications to Tracking and Navigation: Theory Algorithms and Software . Wiley, John \& Sons, 2001.

[39] M. Ester, H.-P. Kriegel, J. Sander, and X. Xu, "A Density-based Algorithm for Discovering Clusters in Large Spatial Databases with Noise," in Proc. of 2nd Int. Conf. on Knowledge Discovery and Data Mining, 1996, pp. 226-231.

[40] D. Schuhmacher, B. T. Vo, and B. N. Vo, "A Consistent Metric for Performance Evaluation of Multi-Object Filters," IEEE Trans. on Signal Processing, vol. 56, no. 8, pp. 3447-3457, Aug. 2008.

[41] T. Li, F. D. la Prieta Pintado, J. M. Corchado, and J. Bajo, "Multi-source homogeneous data clustering for multi-target detection from cluttered background with misdetection," Applied Soft Computing, vol. 60, pp. 436 - 446, 2017.

[42] T. Li, J. M. Corchado, S. Sun, and J. Bajo, "Clustering for filtering: Multi-object detection and estimation using multiple/massive sensors," Information Sciences, vol. 388-389, pp. 172 - 190, 2017.

[43] M. Schikora, A. Gning, L. Mihaylova, D. Cremers, and W. Koch, "Box-Particle Probability Hypothesis Density Filtering," IEEE Trans. on Aerospace and Electronic Systems, vol. 50, no. 3, pp. 1660-1672, July 2014.

[44] H. Haj Chadé, A. Gning, F. Abdallah, I. Mougharbel, and S. Julier, "Non Parametric Distributed Inference in Sensor Networks Using Box Particles Messages," Mathematics in Computer Science, vol. 8, no. 3-4, pp. 455-478, Aug. 2014.

[45] L. Yan, X. R. Li, Y. Xia, and M. Fu, "Modeling and estimation of asynchronous multirate multisensor system with unreliable measurements," IEEE Transactions on Aerospace and Electronic Systems, vol. 51, no. 3, pp. 2012-2026, July 2015.

[46] _ "Optimal sequential and distributed fusion for state estimation in cross-correlated noise," Automatica, vol. 49, no. 12, pp. 3607 - 3612, 2013.

[47] N. Petrov, L. Mihaylova, A. Gning, and D. Angelova, "A Novel Sequential Monte Carlo Approach for Extended Object Tracking Based on Border Parameterisation," in Proc. of 14th Int. Conf. on Information Fusion, 2011, pp. 306-313. 